\title{
THE STRUCTURE OF RATIONAL AND RULED SYMPLECTIC 4-MANIFOLDS
}

\author{
DUSA MCDUFF
}

\section{INTRODUCTION}

In this paper we classify compact symplectic 4-manifolds which contain a symplectically embedded copy $C$ of $S^{2}$ with nonnegative self-intersection number. The classification is almost complete: the one remaining question concerns the uniqueness of blowing up in the symplectic category. The paper was inspired by Gromov's result in [G] that if $(V, \omega)$ is a compact symplectic 4-manifold whose second homology group is generated by a symplectically embedded 2-sphere of self-intersection +1 , then $V$ is $\mathbb{C} P^{2}$ with its usual Kähler structure.

Here are our main results. All manifolds considered will be smooth, compact and, unless specific mention is made to the contrary, without boundary. Throughout, $(V, \omega)$ denotes a symplectic 4-manifold. It will be called minimal if it contains no exceptional curves, that is, symplectically embedded 2-spheres $\Sigma$ with self-intersection number $\Sigma \cdot \Sigma=-1$. We showed in [McD2, Lemma 2.1] that every such curve has a neighbourhood $N_{\varepsilon}$ whose boundary $\left(\partial N_{\varepsilon}, \omega\right)$ may be identified with the boundary $\left(\partial B^{4}(\lambda+\varepsilon), \omega_{0}\right)$ of the ball of radius $\lambda+\varepsilon$ in $\mathbb{C}^{2}$, where $\pi \lambda^{2}=\omega(\Sigma)$ and $\varepsilon>0$ is sufficiently small. Hence the curve $\Sigma$ can be blown down by cutting out $N_{\varepsilon}$ and gluing in the ball $B^{4}(\lambda+\varepsilon)$, with its standard form $\omega_{0}$. It is easy to check that the resulting manifold is independent of the choice of $\varepsilon$, so that there is a well-defined blowing down operation, which is inverse to symplectic blowing up.

Theorem 1.1(i). Every symplectic 4-manifold $(V, \omega)$ covers a minimal symplectic manifold $(\bar{V}, \bar{\omega})$ which may be obtained from $V$ by blowing down a finite collection $\varepsilon$ of disjoint exceptional curves. Moreover, given $\varepsilon$, the induced symplectic form $\bar{\omega}$ on $\bar{V}$ is unique up to isotopy.

Theorem 1.1 also applies to manifold pairs $(V, C)$ where $C$ is a symplectically embedded compact 2-manifold. We will call such a pair minimal if $V-C$

Received by the editors November 28, 1989.

1980 Mathematics Subject Classification (1985 Revision). Primary 53C15, 57R99.

Key words and phrases. Symplectic manifold, 4-manifold, contact structures, pseudo-holomorphic curves, almost complex manifold, blowing up.

Partially supported by NSF grant DMS 8803056. 
contains no exceptional curves. Thus we have

Theorem 1.1(ii). Every symplectic pair $(V, C, \omega)$ covers a minimal symplectic pair $(\bar{V}, C, \bar{\omega})$ which may be obtained by blowing down a finite collection $\varepsilon$ of disjoint exceptional curves in $V-C$. Moreover, given $\varepsilon$, the induced symplectic form $\bar{\omega}$ on $\bar{V}$ is unique up to isotopy $(\mathrm{rel} C)$.

Note. If $A$ is a closed subset of $V$, two symplectic forms $\omega_{0}$ and $\omega_{1}$ are said to be isotopic ( $\operatorname{rel} A$ ) if they can be joined by a family of cohomologous symplectic forms whose restrictions to $A$ are all equal. For further discussion of this notion see Definition 4.3ff.

It is well known that the diffeomorphism type of $\bar{V}$ is not uniquely determined by that of $V$. For example, because $\left(S^{2} \times S^{2}\right) \# \overline{\mathbb{C P}}^{2}$ is diffeomorphic to $\mathbb{C} P^{2} \# \overline{\mathbb{C P}}^{2} \# \overline{\mathbb{C P}}^{2}$, the manifold $V=\left(S^{2} \times S^{2}\right) \# \overline{\mathbb{C P}}^{2}$ may be reduced to $\mathbb{C} P^{2}$ as well as to $S^{2} \times S^{2}$. However, this is essentially the only ambiguity, and we will see that $\bar{V}$ is determined up to symplectomorphism if we fix the homology classes of the curves which are blown down.

Conversely, one can ask to what extent the minimal manifold $(\bar{V}, \bar{\omega})$ determines its blowing up $(V, \omega)$. Since each exceptional curve $\Sigma$ in $(V, \omega)$ corresponds to an embedded ball in $\bar{V}$ of radius $\lambda$, where $\omega(\Sigma)=\pi \lambda^{2}$, this question is related to properties of the space of symplectic embeddings of $\amalg B\left(\lambda_{i}\right)$ into $(\bar{V}, \bar{\omega})$, where $\amalg B\left(\lambda_{i}\right)$ is the disjoint union of the symplectic 4-balls $B\left(\lambda_{i}\right)$ of radius $\lambda_{i}$. We discussed the corresponding question for manifold pairs in [McD2]. We showed there that, if $C \cdot C=1$, and if $V$ is diffeomorphic to $\mathbb{C} P^{2}$ with $k$ points blown up, there is a unique symplectic structure on $(V, C)$ in the cohomology class $a$ if and only if the space of symplectic embeddings of $\amalg B\left(\lambda_{i}\right)$ into $\mathbb{C} P^{2}-\mathbb{C} P^{1}$ is connected, where $\pi \lambda_{i}^{2}, \ldots, \pi \lambda_{k}^{2}$ are the values of $a$ on the exceptional curves in $V$. Unfortunately nothing is known about this space of embeddings per se. In fact, the information we have goes the other way: we proved in [McD2] that the structure on $(V, C)$ is unique when $k=1$, which implies that the corresponding space of embeddings is connected. (Because $\mathbb{C} P^{2} \# \overline{\mathbb{C P}}^{2}$ is ruled, this uniqueness statement is closely related to the results in Theorem 1.3 below.) It is not clear what happens when $k \geq 2$. However, because any two embeddings of $\amalg B\left(\lambda_{i}\right)$ into $(\bar{V}, \bar{\omega})$ are isotopic when restricted to the union $\amalg B\left(\varepsilon_{i}\right)$ of suitably small subballs, any two symplectic forms on $V$ which blow down to $\bar{\omega}$ may be joined by a family of noncohomologous symplectic forms.

Because blowing up and down in the symplectic category involves large balls rather than just points, it is not obvious that the category of symplectic manifolds which we are considering is closed under these operations. However, we will prove

Theorem 1.2. The category of symplectic 4-manifolds $(V, \omega)$ which contain a symplectically embedded copy of $S^{2}$ with nonnegative self-intersection is closed under blowings up and down. It is also closed under perturbations of $\omega$ through 
noncohomologous symplectic forms: in other words, if $\omega_{t}, 0 \leq t \leq 1$, is a family of symplectic forms such that $\left(V, \omega_{0}\right)$ belongs to this category, then $\left(V, \omega_{1}\right)$ does too.

The above results show that the classification problem is essentially reduced to understanding the minimal case. The next ingredient is a result on the structure of symplectic $S^{2}$-bundles (symplectic ruled surfaces).

Theorem 1.3. Let $V$ be an oriented $S^{2}$-bundle $\pi: V \rightarrow M$ over a compact oriented surface $M$ with fiber $F$.

(i) The cohomology class a of any symplectic form on $V$ which is nondegenerate on each fiber of $\pi$ satisfies the conditions:

(a) $a(F)$ and $a^{2}(V)$ are positive, and

(b) $a^{2}(V)>(a(F))^{2}$ if the bundle is nontrivial.

(ii) Any cohomology class $a \in H^{2}(V ; \mathbb{R})$ which satisfies the above conditions may be represented by a symplectic form $\omega$ which is nondegenerate on each fiber of $\pi$. Moreover, this form is unique up to isotopy.

The existence statement in (ii) above is well known. It is obvious if the bundle is trivial. If it is nontrivial, one can think of $V$ as the suspension of a circle bundle of Euler class 1 with the corresponding $S^{1}$-action, and can then provide $V$ with an invariant symplectic form in any class $a$ which satisfies (i) $(a, b)$ since these conditions correspond to requiring that $a$ be positive on each of the two fixed point sets of the $S^{1}$-action. (See [Au].)

The other statements are more delicate. Consider first the case when the base manifold $M$ is $S^{2}$. Gromov showed in [G] that any symplectic form on $S^{2} \times S^{2}$ which admits symplectically embedded spheres in the classes $\left[S^{2} \times \mathrm{pt}\right]$ and [pt $\left.\times S^{2}\right]$ is isotopic to a product (or split) form. (In fact, Gromov assumed that the form has equal integrals over the two spheres, but it is not hard to remove this condition.) A corresponding uniqueness result when $V=\mathbb{C} P^{2} \# \overline{\mathbb{C P}}^{2}$ (which is the nontrivial $S^{2}$-bundle over $S^{2}$ ) was proved in [McD2]. This result requires the existence of just one symplectically embedded sphere, but it must be in the class of a section of self-intersection 1, not of the fiber. Gromov showed that this hypothesis also implies that there must be a symplectically embedded sphere in the class of the blown-up point, which, as we will see below, is equivalent to condition (i)(b). In the present situation, we have less information since we start with only one symplectically embedded sphere, the fiber. Following an idea of Eliashberg's, we can construct a symplectic section of $\pi$ (i.e., a section on which the symplectic form $\omega$ does not vanish) and so reduce to the previously considered case. In the process, we have to change the form $\omega$ by adding $\pi^{*}(\sigma)$ where $\sigma$ is a 2-form on $M$ such that $\sigma(M)>0$. We then use the theory of holomorphic curves to show that if $\omega_{t}$, $0 \leq t \leq 1$, is a family of (noncohomologous) symplectic forms on $V$ such that $\omega_{1}$ admits a symplectic section, then so does $\omega_{0}$. When $M$ is an arbitrary 
Riemann surface, these arguments must be supplemented by some cutting and pasting in order to reduce to the case when $M=S^{2}$.

We can now state the classification theorem for minimal symplectic pairs. By analogy with the complex case, we will sometimes call a symplectic image of $S^{2}$ in $V$ a "rational curve".

Theorem 1.4. Let $(V, C, \omega)$ be a minimal symplectic 4-dimensional pair where $C$ is a rational curve with self-intersection $C \cdot C=p \geq 0$. Then $(V, \omega)$ is symplectomorphic either to $\mathbb{C} P^{2}$ with its usual Kähler form or to a symplectic $S^{2}$-bundle over a compact surface $M$. Further, this symplectomorphism may be chosen so that it takes $C$ either to a complex line or quadric in $\mathbb{C} P^{2}$, or to a fiber of the $S^{2}$-bundle, or (if $M$ is $S^{2}$ ) to a section of this bundle.

From this, it is easy to prove

Corollary 1.5. (i) If $(V, C, \omega)$ is as above, the diffeomorphism type of the pair $(V, C)$ is determined by $p$ provided that $p \neq 0,4$.

(ii) When $p=4$, there are two possibilities for $(V, C)$ : it can be either $\left(\mathbb{C} P^{2}, Q\right)$ or $\left(S^{2} \times S^{2}, \Gamma_{2}\right)$, where $Q$ is a quadric and where $\Gamma_{2}$ is the graph of a holomorphic self-map of $S^{2}$ of degree 2. When $p=0, C$ is a fiber of $a$ symplectic $S^{2}$-bundle.

(iii) $(V, C, \omega)$ is determined up to symplectomorphism by the cohomology class of $\omega$.

Corollary 1.6. A minimal symplectic 4-manifold $(V, \omega)$ which contains a rational curve $C$ with $C \cdot C>0$, is symplectomorphic either to $\mathbb{C} P^{2}$ or to $S^{2} \times S^{2}$ with the standard form.

The main tool in the proof of Theorem 1.4 is the following homological version of the adjunction formula. We will suppose that $J$ is a $C^{\infty}$-almost complex structure on $V$ with first Chern class $c$. If $C$ is a $J$-holomorphic curve (see $\S 2$ ), we define its virtual genus $g(C)$ to be

$$
g(C)=1+\frac{1}{2}(C \cdot C-c(C)) .
$$

In [McD4] we show that if $C$ is the $J$-holomorphic image of a Riemann surface of genus $g$ then $g(C) \geq g$, with equality if and only if $C$ is embedded. This is a homological criterion for a curve to be embedded, and using it, we prove Theorem 1.4 by showing that $V$ must contain an embedded $J$-simple curve of self-intersection +1 or 0 . ( $J$-simple curves do not decompose, so that their moduli space is compact.) It then follows by arguments of Gromov that $V$ is $\mathbb{C} P^{2}$ in the former case and a symplectic $S^{2}$-bundle in the latter.

Thus, the symplectic 4-manifolds under consideration behave in much the same way as complex surfaces. In particular, the analogue of Noether's rationality criterion holds: if $(V, \omega)$ contains an embedded rational curve $C$ with $C \cdot C>0$, then $(V, \omega)$ is birationally equivalent to $\mathbb{C} P^{2}$ with its standard structure. Here "birational equivalence" means that one can get from 
one manifold to the other by a sequence of symplectic blowings up and down. Guillemin and Sternberg show in [GS] that this relation can be thought of as a special kind of symplectic cobordism. Observe that, by Theorem 1.2, our class of manifolds is closed under birational equivalence.

Note. Given an arbitrary symplectic 4-manifold one can always blow up some points to create a manifold $(V, \omega)$ which contains a symplectically embedded 2-sphere with an arbitrary negative self-intersection number. Hence, the existence of such a 2-sphere gives no information on the structure of $V$.

Corollary 1.5 may be understood as a statement abut the uniqueness of symplectic fillings of certain contact manifolds. Indeed, consider an oriented $(2 n-1)$-dimensional manifold $\Delta$ with closed 2-form $\sigma$. We will say that $(\Delta, \sigma)$ has contact type if there is a positively oriented contact form $\alpha$ on $\Delta$ such that $d \alpha=\sigma$. It is easy to check that the contact structure thus defined is independent of the choice of $\alpha$. Following Eliashberg [E], we say that the symplectic manifold $(Z, \omega)$ fills $(\Delta, \sigma)$ if there is a diffeomorphism $f: \partial Z \rightarrow \Delta$ such that $f^{*}(\sigma)=\omega \mid \partial Z$. Further, the filling $(Z, \omega)$ is said to be minimal if $Z$ contains no exceptional curves in its interior.

As Eliashberg points out, information on symplectic fillings provides a way to distinguish between contact structures: if one constructs a filling of $\left(\Delta, \sigma_{2}\right)$ which does not have a certain property which one knows must be possessed by all fillings of $\left(\Delta, \sigma_{1}\right)$, then the contact structures on $\Delta$ defined by $\sigma_{1}$ and $\sigma_{2}$ must be different. In particular, it is interesting to look for manifolds of contact type which have unique minimal fillings. Obvious candidates are the lens spaces $L_{p}, p>1$, which are obtained as the quotients of $S^{3} \subset \mathbb{C}^{2}$ by the standard diagonal action of the cyclic subgroup $\Gamma_{p} \subset S^{1}$ of order $p$ on $\mathbb{C}^{2}$, and whose 2-form $\sigma$ is induced by $\omega_{0}$. It is not hard to see that if $(Z, \omega)$ fills $\left(L_{p}, \sigma\right)$ we may quotient out $\partial Z=L_{p}$ by the Hopf map to obtain a rational curve $C_{p}$ with self-intersection $p$ in a symplectic manifold $(V, \omega)$ without boundary. Hence Corollary 1.5 implies

Theorem 1.7. The lens spaces $L_{p}, p \geq 1$, all have minimal symplectic fillings. If $p \neq 4$, minimal fillings $(Z, \omega)$ of $\left(L_{p}, \sigma\right)$ are unique up to diffeomorphism, and up to symplectomorphism if one fixes the cohomology class $[\omega]$. However, $\left(L_{4}, \sigma\right)$ has exactly two nondiffeomorphic minimal fillings.

In higher dimensions, one cannot hope for such precise results. However, in dimension 6 there are certain contact-type manifolds (such as the standard contact sphere $S^{5}$ ) which impose conditions on any filling $(Z, \omega)$, even though they may not dictate the diffeomorphism type of minimal fillings. In dimensions $>6$, one must restrict to "semi-positive" fillings to get analogous results. See [McD5].

In order to make this paper reasonably self-contained we have summarized what we need of the theory of pseudo-holomorphic curves in $\S 2$. The rest of this section details various consequences of the adjunction formula. Theorem 1.1 
on blowing down and minimality is proved in $\S 3$. The structure of symplectic $S^{2}$-bundles is examined in $\S 4$, while $\S 5$ contains proofs of the classification Theorems 1.4 and 1.7 , as well as Theorem 1.2.

\section{J-HOLOMORPHIC CURVES IN 4-MANIFOLDS}

This section establishes the basic results about $J$-holomorphic curves on symplectic 4-manifolds which we will need later. For the convenience of the reader we will begin with a brief summary of Gromov's theory. For more details see [McD1, §4; McD3].

First recall that an almost complex structure $J$ on a symplectic manifold $(V, \omega)$ is said to be $\omega$-tame if $\omega$ is positive on all $J$-complex lines in $T V$. We will denote the Sobolev space of all $H^{s}$-smooth $\omega$-tame $J$ by $\mathcal{J}(\omega)$, where $s$ is suitably large. It is easy to check that $\mathscr{J}(\omega)$ is nonempty and contractible. Given a homology class $A \in H_{2}(V, \mathbb{Z})$ and an $\omega$-tame $J$, a parametrized $J$ holomorphic A-curve (or $A$-curve for short) is a map $f$ from a Riemann surface $\left(\Sigma_{g}, \bar{J}\right)$ to $V$, which represents the class $A$, and is $J$-holomorphic in the sense that $d f \circ \bar{J}=J \circ d f$. If we need to emphasize the role of $\bar{J}$ we will call such $f$ $\bar{J}$ - $J$-holomorphic. Further, unless specific mention is made to the contrary, we will assume that $f$ is not a multiple covering, i.e., it does not factor as $f^{\prime} \circ \gamma$, where $\gamma: \Sigma_{g} \rightarrow \Sigma_{g}$ is a $\bar{J}$-holomorphic self-map of $\Sigma_{g}$. (According to [McD1, 4.4] this will be the case provided that $f$ is somewhere injective, i.e., there is some $z \in \Sigma_{g}$ such that $f^{-1}(f(z))=\{z\}$.) We define the moduli space $\mathscr{M}_{A, g}$ by setting

$$
\mathscr{M}_{A, g}=\left\{(f, \bar{J}, J) \in F \times \mathscr{T}_{g} \times \mathscr{J}(\omega): f \text { is } \bar{J} \text { - } J \text {-holomorphic }\right\}
$$

where $F$ is a suitable Sobolev space of somewhere injective maps $\Sigma_{g} \rightarrow V$, and $\mathscr{T}_{g}$ is the genus $g$ Teichmüller space. There is a projection operator $P_{A}: \mathscr{M}_{A, g} \rightarrow \mathscr{J}(\omega)$ defined by $(f, \bar{J}, J) \mapsto J$, and we write $M_{p}\left(J, \mathscr{T}_{g}, A\right)$ for the inverse image $P_{A}^{-1}(J)$. Thus $M_{p}\left(J, \mathscr{T}_{g}, A\right)$ is the space of all parametrized $J$-holomorphic $A$-curves of genus $g$. If the genus $g$ is zero, then the curve is said to be rational and we suppress $g$ and $\mathscr{T}_{g}$ from the notation.

(2.1) Fredholm property. The main results are that $\mathscr{M}_{A, g}$ is a Hilbert manifold and that the projection operator $P_{A}: \mathscr{M}_{A, g} \rightarrow \mathcal{J}(\omega)$ is Fredholm. (The latter result holds because the linearization $d P_{A}$ of $P_{A}$ is a generalized CauchyRiemann operator, and so is elliptic.) This means that for generic $J$ in $\mathscr{J}(\omega)$, $M_{p}\left(J, \mathscr{T}_{g}, A\right)$ is a manifold of dimension Ind $P_{A}=\operatorname{dim} \mathscr{T}_{g}+2(c(A)+n(1-g))$, where $2 n=\operatorname{dim} V, g=$ genus $\Sigma_{g}$, and $c(A)$ is the value taken by the first Chern class of $V$ on $A$. This was proved in [McD1] for rational curves, and the proof goes over without essential change for curves of higher genus. The formula for the index is discussed in [G, (2.1.A)].

(2.2) The compactness theorem (see [G, 1.5.B; W]). In the rational case, this implies that the quotient space $M(J, A)=M_{p}(J, A) / G$ of unparametrized 
rational curves is compact whenever suitable assumptions are made about the class $A$. (Here $G$ is the reparametrization group $\operatorname{PSL}(2, \mathbb{C})$.) In particular, $M(J, A)$ is compact when $A$ is $J$-simple, which means that $A$ does not split as a sum $A_{1}+\cdots+A_{k}$ of classes all of which can be represented by $J$-holomorphic curves. (For a more precise definition in dimension 4, see $\S 5$.) The class $A$ is called simple if it is $J$-simple for all $\omega$-tame $J$.

If $A$ is not simple, then $M(J, A)$ is not in general compact. However Gromov and Wolfson show that if $J_{i}$ is a sequence in $\mathscr{J}(\omega)$ which converges to $J_{0}$ in $\mathscr{J}(\omega)$ in the $C^{k+\alpha}$-topology, where $k \geq 2$, then any sequence $C_{i}$ of rational $J_{i}$-holomorphic curves contains a subsequence which converges weakly to a rational $J_{0}$-holomorphic curve (which might be multiply-covered) or to a cusp-curve $C$, provided only that $\omega\left(C_{i}\right)$ is uniformly bounded. (A rational cusp-curve is a connected union of two or more rational curves-the "original" curve plus some "bubbles"-some of which may coincide or even be multiplycovered. The notion of weak convergence is quite complicated, but it implies convergence in the Hausdorff topology for subsets of $V$.) As we have done here, we will often denote an unparametrized curve $\operatorname{Im} f$ by $C$.

When considering curves of higher genus there is another source of noncompactness since the domain $(\Sigma, \bar{J})$ may not stay within a bounded region in Teichmüller space. However, a statement analogous to that in the paragraph above still holds; see [W].

(2.3) Smoothness. Whenever the space of (unparametrized) curves under consideration is compact, the set of generic almost complex structures is open and dense in $\mathscr{J}(\omega)$, and so we may choose our almost complex structures to be $C^{\infty}$. This means that all the maps $f$ in $M_{p}\left(J, \mathscr{T}_{g}, A\right)$ are $C^{\infty}$, and allows us to apply the results in (2.1)(iv) and (2.1)(v) below. In fact, by being a little more careful about setting up the Fredholm theory, one need only consider $C^{\infty}$-smooth $J$; see [McD3, §2].

(2.4) Chern class. In dimension 4 , a curve $C$, which is the $J$-holomorphic image of a Riemann surface $\Sigma_{g}$ of genus $g$ for generic $J$, must have $c(C) \geq$ $1-g$. More precisely, given $K>0$, define $\mathscr{U}(K, g)$ to be the subset of $\mathscr{J}(\omega)$ consisting of all $J$ such that, for each homology class $B$ such that $c(B) \leq-g$ and $\omega(B) \leq K$, there are no $J$-holomorphic images of $\Sigma_{g}$ in class $B$. Then the argument in [McD2, (3.1)(iv)] shows that $\mathscr{U}(K, g)$ is open, dense, anc pathconnected. (The main point here is that if $c(B)<1-g$, where $g>1$, then Ind $P_{B}$ is negative so that no $J$-holomorphic $B$-curve of genus $g$ is regular. Therefore, for generic $J$ there are no $J$-holomorphic $B$-curves. A similar conclusion follows when $g=0,1$ since in this case the index of $P_{B}$ will be less than the dimension of the group of holomorphic self-maps of $\Sigma_{g}$.)

(2.5) Positivity of intersections (see [G1, 2.1. $\left.\mathrm{C}_{2} ; \mathrm{McD} 4\right]$ ). Assume that $J$ is $C^{\infty}$. Then, an intersection point of two distinct $J$-holomorphic curves $C$ and $C^{\prime}$ always occurs with positive orientation. Moreover, if the curves $C$ and $C^{\prime}$ 
intersect at a point $x$ which is a singular point of $C$, or if the intersection is not transverse, then $x$ contributes a number $k_{x}>1$ to the algebraic intersection number $C \cdot C^{\prime}$. Thus, $C \cdot C^{\prime}=0$ if and only if $C$ and $C^{\prime}$ are disjoint; and $C \cdot C^{\prime}=1$ if and only if $C$ and $C^{\prime}$ meet exactly once transversally, and at a point which is nonsingular on both curves. Further, if two distinct classes $A$ and $B$ are realized by $J$-holomorphic curves for the same $J$, then $A \cdot B \geq 0$.

(2.6) The virtual genus of a curve. Assume that $J$ is $C^{\infty}$. Then, the virtual genus $g(C)$ of a rational $J$-holomorphic curve is defined to be

$$
g(C)=1+\frac{1}{2}(C \cdot C-c(C)) .
$$

We show in [McD4, Theorem 1.3] that $g(C)$ is a nonnegative integer which equals 0 if and only if $C$ is embedded. (This can be considered as a homological version of the classical adjunction formula.) A similar result is true for an arbitrary curve, but we shall not need it here.

(2.7) Regularity. We next discuss a criterion for an embedded curve $C=\operatorname{Im} f$ to be regular, i.e., for the triple $(f, \bar{J}, J)$ to be a regular point for the operator $P_{A}$ of (2.1). If $C$ is rational and $J$ is integrable near $C$, it follows from [McD3, Lemma 2.3.4] that the curve is regular if and only if the first Chern number $c_{\nu}$ of the normal bundle of $C$ in $V$ is greater than -2 . We now prove the analogue of this result for higher genus. This question is discussed implicitly by Gromov in [G, 2.1], but he never quite makes clear the role of $\mathscr{T}_{g}$. Also, the formula which he gives for $c^{*}$ in 2.1. $C_{1}$ should read $c^{*}=2(q-1)-c_{\nu}$.

Lemma 2.8. If $f: \Sigma_{g} \rightarrow V$ is an embedded $\bar{J}$-J-holomorphic curve of genus $g$, such that $J$ is integrable near $\operatorname{Im} f$, then $(f, \bar{J}, J)$ is a regular point for the operator $P_{A}$ if and only if the Dolbeault cohomology group $H^{1}\left(\Sigma, \nu_{\Sigma}\right)$ vanishes, where $\nu_{\Sigma}$ is the pull-back to $\Sigma_{g}$ of the normal bundle to $\operatorname{Im} f$ in $V$, considered as a $\bar{J}$-holomorphic complex line bundle. Thus the condition is $c_{\nu}>2(g-1)$.

Proof. If $\bar{J}$ is kept fixed, then it is shown in [McD1, §4.1] that $d P_{A}$ may be identified with the usual $\bar{\partial}$-operator from the smooth sections $\Lambda^{0,0}\left(\Sigma_{g}, E\right)$ of the bundle $E=f^{*}(T V)$ to the $(0,1)$-forms $\Lambda^{0,1}\left(\Sigma_{g}, E\right)$. Since in the present situation $\bar{J}$ is allowed to vary in $\mathscr{T}_{g}$, the operator $d P_{A}$ which we must now consider is the sum of this $\bar{\partial}$-operator with an operator from $T_{J}\left(\mathscr{T}_{g}\right)$ to $\Lambda^{0,1}\left(\Sigma_{g}, E\right)$. It is easy to check that this latter operator has image equal to $\Lambda^{0,1}\left(\Sigma_{g}, T \Sigma_{g}\right)$. Hence Coker $d P_{A}$ is isomorphic to $H^{1}\left(\Sigma_{g}, \nu_{\Sigma}\right)$, and hence, via Kodaira-Serre duality, to $H^{0}\left(\Sigma_{g}, \nu_{E}^{*} \otimes K_{\Sigma}\right)$, where $K_{\Sigma}$ is the canonical bundle over $\Sigma_{g}$. It is well known that a holomorphic line bundle has no holomorphic sections if its first Chern class is negative. But $c_{1}\left(\nu_{E}^{*} \otimes K_{\Sigma}\right)=-c_{\nu}+2(g-1)$. Hence the results.

We now use (2.6) to obtain information on the weak limit $S$ of a sequence of rational $J$-holomorphic embedded curves. Observe that, since the virtual 
genus of the latter curves is zero, $S$ must satisfy the equation $S \cdot S=c(S)-2$. Throughout the following discussion, we will assume without further mention that all curves are rational and that $J$ is $C^{\infty}$. We begin with a simple lemma.

Lemma 2.9. Suppose that $A$ and $B$ are elements of $H_{2}(V, \mathbb{Z})$ such that $A \cdot A=$ $c(A)-2, B \cdot B \geq c(B)-2$, and $A=k B$ for some integer $k \geq 2$. Then, if $c(A) \geq 1, k=2$ and $1=B \cdot B=c(B)-2$.

Proof. Since $A \cdot A=k^{2} B \cdot B \geq-1$, both $A \cdot A$ and $B \cdot B$ must be nonnegative. If $A \cdot A=B \cdot B=0$, then $c(A)=2, k=2$, and $c(B)=1$. But this is impossible since $B \cdot B-c(B)$ is even by (2.6). Thus $A \cdot A$ and $B \cdot B$ are positive, and we have

$$
\begin{aligned}
A \cdot A & =k^{2} B \cdot B=\left(k^{2}-k\right) B \cdot B+k B \cdot B \\
& \geq\left(k^{2}-k\right)+k(c(B)-2) \geq k c(B)-2=c(A)-2
\end{aligned}
$$

because $k^{2}-3 k+2 \geq 0$ if $k>1$. Note that equality can occur here only if $k=2$ and $B \cdot B=1$.

Corollary 2.10. If $C$ is a multiply-covered curve which is the limit of a sequence of rational J-holomorphic embedded curves, then $C$ is a double covering of an embedded curve with self-intersection +1 .

By the compactness theorem, a sequence of rational $J$-holomorphic embedded curves abuts either on a curve (possibly multiply-covered) or on a cuspcurve, which, by definition, contains at least two components. These components are rational, some of them might coincide and some might even be multiply-covered. We now investigate the possibilities when $S$ is a cusp-curve. We remind the reader that the word "curve" below always denotes the image of a somewhere injective $J$-holomorphic map. If this map might be a multiple covering we use the word "component". Recall further that an exceptional curve is an embedded rational curve of self-intersection number -1 . The homology class $\left[S_{i}\right]$ of a component $S_{i}$ will also be denoted by $A_{i}$.

Proposition 2.11. Let $S_{1}, \ldots, S_{m}$ be the components of a J-holomorphic cuspcurve $S$ which satisfies the equation $S \cdot S=c(S)-2$. We suppose that each component $S_{i}$ is (a multiple covering of ) a regular curve and that $c\left(A_{i}\right) \geq 1$ for all $i$.

(i) If $A_{i} \cdot A_{i} \geq 1$ for some $i$, then $S_{i}$ is an embedded curve, and $A_{i} \neq A_{j}$ for any $j \neq i$ except possibly if $m=2$ and $A_{i} \cdot A_{i}=1$.

(ii) If $A_{i} \cdot A_{i}=0$ for some $i$, then $S_{i}$ is a $k$-fold cover of an embedded curve for some $k \geq 1$.

(iii) If $A_{i} \cdot A_{i}<0$ for some $i$, then either $S_{i}$ is an exceptional curve and $A_{i}=A_{j}$ for at most one $j \neq i$, or $S_{i}$ is a double cover of an exceptional curve and $A_{i} \neq c A_{j}$ for any $j \neq i$ or $c>0$. Further $A_{i} \cdot[S]=0$ except in the case when $S_{i}$ is an exceptional curve distinct from all other $S_{j}$. 
Proof. The proof of Lemma 2.9 shows that if $S_{i}$ is a $k$-fold cover of a curve $T$ such that $T \cdot T \geq 1$, then

$$
S_{i} \cdot S_{i} \geq c\left(S_{i}\right)-2
$$

Note that equality can occur here only if $k=2$ and $T \cdot T=1$.

Because $c\left(S_{i}\right) \geq 1$ for all $i,(2.6)$ implies that no component can be a (multiple cover of a) curve with self-intersection $<-1$, and that any $J$-holomorphic curve $T$ with self-intersection -1 or 0 must be embedded. This proves (ii). Next, observe that, because all the curves under consideration are regular by hypothesis, any curve $T$ with $T \cdot T=0$ is part of a (local) 2-parameter family of embedded $J$-holomorphic [T]-curves. Hence, we may replace any component of $S$ which is a $k$-fold cover of $T$ by $k$ disjoint copies of $T$ (which do not coincide with any other component). Note that this does not disconnect $S$. For, because $S$ has more than one component, either there is some component $S_{i}$ such that $S_{i} \cdot T>0$ in which case $S_{i}$ will intersect all the copies of $T$, or all the components of $S$ are multiple coverings of $T$. But the proof of Lemma 2.9 shows that the latter case does not occur. Thus we may assume that all the components with zero self-intersection are distinct embedded curves and therefore satisfy (\#).

We next arrange that none of the homology classes $A_{i}$ of the components $S_{i}$ with $S_{i} \cdot S_{i} \neq 0$ are multiples of each other. For if $A_{i}=c A_{j}$ for some $c \geq 1$, we may replace the components $S_{i}$ and $S_{j}$ by a single multiply-covered curve without disconnecting $S$ or changing $[S]$. Since this operation reduces the number $m$ of components, it is possible that the result consists of a single multiply-covered curve $k T$. But then Corollary 2.10 implies that we started with a cusp-curve which had exactly two homologous embedded components of self-intersection +1 . Since this possibility is covered in (i), the proposition holds in this case.

Now suppose that $m>1$, that $A_{i} \neq c A_{j}$ when $i \neq j$ and $A_{i} \cdot A_{i} \neq 0$, and that all the components of $S=S_{1} \cup \cdots \cup S_{m}$ satisfy (\#). Since $S \cdot S=c(S)-2$, we have

$$
\sum_{i} S_{i} \cdot S_{i}+2 \sum_{i<j} S_{i} \cdot S_{j}=\sum_{i} c\left(S_{i}\right)-2
$$

Also, because $S$ is connected and the $S_{i}$ are distinct, there must be at least $m-1$ pairs of components $S_{i}, S_{j}$ which intersect. Thus $\sum_{i<j} S_{i} \cdot S_{j} \geq m-1$, and so

$$
\sum_{i} S_{i} \cdot S_{i} \leq \sum_{i}\left(c\left(S_{i}\right)-2\right)
$$

Therefore, by (\#), we must have $S_{i} \cdot S_{i}=c\left(S_{i}\right)-2$ for each $i$. Further, $\sum_{i<j} S_{i} \cdot S_{j}=m-1>0$ which means that none of the components $S_{i}$ with $S_{i} \cdot S_{i}>0$ can be multiple covers, and so they are all embedded curves in distinct homology classes as required by proposition (i). 
It remains to consider the case when some of the $S_{i}$ are $k_{i}$-fold covers of curves $R_{i}$ of negative self-intersection so that (\#) does not hold for these $i$. We remarked above that the $R_{i}$ must be embedded and satisfy $R_{i} \cdot R_{i}=-1$. Let us reorder the $S_{i}$ so that $S=k_{1} R_{1} \cup \cdots \cup k_{r} R_{r} \cup S_{r+1} \cup \cdots \cup S_{m}$ where $k_{p}>1$ for $p \leq r$, and where the $R_{p}$ and $S_{i}$, for $i>r$, satisfy (\#). Then, because $R_{p} \cdot S \geq 0$, for each $p$,

$$
\sum_{i>r} R_{p} \cdot S_{i}+\sum_{q \neq p} R_{p} \cdot k_{q} R_{q} \geq k_{p}
$$

Thus

$$
\begin{aligned}
S \cdot S= & -\sum k_{p}^{2}+\sum_{r<i} S_{i} \cdot S_{i} \\
& +2\left(\sum_{i>r, p} k_{p} R_{p} \cdot S_{i}+\sum_{p<q} k_{p} R_{p} \cdot k_{q} R_{q}+\sum_{r<i<j} S_{i} \cdot S_{j}\right) \\
\geq & -\sum k_{p}^{2}+\sum_{r<i} S_{i} \cdot S_{i}+2\left(\sum k_{p}^{2}+m-1-\sum k_{p}\right) .
\end{aligned}
$$

The second inequality arises as follows. Because $S$ is connected, there must be at least $m-1$ distinct pairs from $\left\{R_{1}, \ldots, R_{r}, S_{r+1}, \ldots, S_{m}\right\}$ which intersect. Clearly, $S \cdot S$ will be smallest if $R_{p} \cdot R_{q}=0$ for $p \neq q$ since the contribution of each such term to $S \cdot S$ is $k_{p} k_{q} R_{p} \cdot R_{q}$. Then, (*) implies that $\sum_{i>r} R_{p} \cdot S_{i} \geq k_{p}$. Hence, the lowest possible estimate for $S \cdot S$ arises if there are exactly $k_{p}$ distinct intersections of the $S_{i}$ with $R_{p}$ (which contributes $k_{p}^{2}$ to the sum), and if the remaining $\left(m-1-\sum k_{p}\right)$ intersections occur as $S_{i} \cap S_{j}$ for $r<i<j$. Since $S \cdot S=c(S)-2$ and $c\left(R_{p}\right)=1$ we find

$$
\sum_{r<i} S_{i} \cdot S_{i} \leq \sum_{r<i}\left(c\left(S_{i}\right)-2\right)-\sum\left(k_{p}^{2}-3 k_{p}+2\right) .
$$

Since $\sum\left(k_{p}^{2}-3 k_{p}+2\right) \geq 0$, this is possible only if all the inequalities are equalities. As before, this means that each $S_{i}, i>r$, is embedded. Further, the $R_{p}$ are disjoint, and, for each $p, R_{p} \cdot S=0$ and $k_{p}=2$. Thus condition (iii) is satisfied.

\section{BLOWING DOWN AND MINIMALITY}

We now turn to the proof of Theorem 1.1, which states that any compact manifold $(V, \omega)$ covers a minimal manifold $(\bar{V}, \bar{\omega})$ which may be obtained by blowing down a set of disjoint exceptional curves simultaneously. Now, the definition of exceptional curve $\Sigma$ was purely symplectic: we required that $\Sigma$ be a symplectically embedded 2-sphere in a class $E$ for which $E \cdot E=-1$. Since each blowing down operation reduces the rank of $H_{2}(V, \mathbb{Z})$, there evidently is a finite sequence of blowing down operations which reduces $V$ to a minimal manifold. However, there seems to be no elementary way of proving that one blowing down will suffice. 
The problem is that, even if one blows down a maximal collection of disjoint exceptional curves, it is not clear that the resulting manifold $(\bar{V}, \bar{\omega})$ is minimal. In fact, the symplectic form $\omega$ is constructed as a iarge perturbation of $\bar{\omega}$, and it is not obvious how to lift an exceptional curve on $\bar{V}$ to one on $V$. One way of getting around this difficulty is to note that there are tame almost complex structures $J$ and $\bar{J}$ on $V$ and $\bar{V}$, respectively, such that the blowing down map is holomorphic. Then, provided that exceptional curves may be assumed to be holomorphic, one can lift them from $\bar{V}$ to $V$.

Here are the details of this argument. The first lemma describes the holomorphic representatives of an exceptional curve $\Sigma$. Recall from (2.2) that a cusp-curve is a connected union of two or more $J$-holomorphic curves, some of which may be multiply-covered. We write (cusp-)curve to denote something which is either a curve or a cusp-curve. Let $\mathscr{U}$ be the subset of $\mathscr{J}(\omega)$ defined in (2.4) with $K \geq \omega(\Sigma)$, and write $\mathscr{U}_{\infty}$ for the subset of $\mathscr{U}$ consisting of elements which are $C^{\infty}$-smooth.

Lemma 3.1. Let $\Sigma$ be an exceptional curve in class $E$. For each $\omega$-tame $J$ there is a unique $J$-holomorphic $E$-(cusp-)curve $\Sigma_{J}$, which varies smoothly with $J$. Moreover, it is a curve if $J \in \mathscr{U}$, and is embedded if $J \in \mathscr{U}$ is sufficiently close to $\mathscr{U}_{\infty}$.

Proof. By the symplectic neighbourhood theorem, $\Sigma$ has a neighbourhood $N$ which is symplectomorphic to a neighbourhood of the zero section in the canonical line bundle $L \rightarrow \mathbb{C} P^{1}$. Thus $N$ has an $\omega$-tame integrable complex structure for which $\Sigma$ is holomorphic, and we may extend this to get an $\omega$-tame almost complex structure $J_{1}$ on $V$. By (2.7), $\Sigma$ is regular. Since there can be at most one $J_{1}$-holomorphic $E$-curve by (2.5), $J_{1}$ must be a regular value for $P_{E}$. It follows that $E$ has a $J$-holomorphic representative $\Sigma_{J}$ for each $J$ near $J_{1}$, and in particular for some $P_{E}$-regular element $J \in \mathscr{U}_{\infty}$. Since the curves $\Sigma_{J}$ are all isotopic if $J$ is sufficiently close to $J_{1}$, and since the image of $J \in \mathscr{U}_{\infty}$ under small isotopies remains in $\mathscr{U}_{\infty}$, we may in fact suppose that $J_{1}$ itself lies in $\mathscr{U}_{\infty}$.

Next, observe that $E$ is $J$-simple for $J \in \mathscr{U}$. For, if $C_{1}, \ldots, C_{k}$ are the components of any cusp-curve in class $E$, then $1=c(E)=\Sigma_{i} c\left(C_{i}\right)$ which implies that at least one of the $c\left(C_{i}\right)$ is $<1$. But this is impossible by definition of $\mathscr{U}$. Further, because $E \cdot E=-1,(2.5)$ implies that for any $J$ there can be at most one (unparametrized) $J$-holomorphic $E$-curve.

Now, consider two regular values $J_{1}$ and $J_{2}$ for $P_{E}$ in $\mathscr{U}$, and let $\alpha$ be a path in $\mathscr{U}$ joining them which is transverse to $P_{E}$. Because $E$ is $J$-simple for each $J \in \alpha, P_{E}^{-1}(\alpha) / G$ is a compact cobordism between the discrete sets $M_{p}\left(J_{1}, E\right) / G$ and $M_{p}\left(J_{2}, E\right) / G$. But the number of points in $M_{p}(J, E) / G$ counted $\bmod 2$ is a cobordism invariant. Hence, there must be exactly one $J$ holomorphic $E$-curve for each $P_{E}$-regular $J \in \mathscr{U}$. Gromov's compactness theorem then implies that for every $J \in \mathscr{J}(\omega)$, there is exactly one $J$-holomorphic $E$-(cusp-)curve, which must be a curve when $J \in \mathscr{U}$. Further, if $J \in \mathscr{U}_{\infty}$, this 
curve is embedded by (2.6). Moreover, $\Sigma_{J}$ must vary smoothly with $J$ by uniqueness, which means in particular that it is embedded for $J$ sufficiently close to $\mathscr{U}_{\infty}$.

Note that each collection of disjoint exceptional curves contains at most $r$ elements where $r$ is the rank of $H_{2}(V ; \mathbb{Z})$. Hence we may choose such a collection $\mathscr{E}$ with the maximal number of elements. Let $\left\{E_{1}, \ldots, E_{k}\right\}$ be the set of homology classes represented by the elements of $\mathscr{E}$, and let $K=$ $\sup \left\{\omega\left(E_{i}\right): i=1, \ldots, k\right\}$.

Lemma 3.2. Given $\mathscr{E}$ as above, one can construct the blowing down map $\pi$ : $(V, \omega) \rightarrow(\bar{V}, \bar{\omega})$ so that it is holomorphic with respect to tame almost complex structures $J_{1}$ and $\bar{J}_{1}$ on $V$ and $\bar{V}$ respectively.

Proof. Let $J_{1}$ be an $\omega$-tame almost complex structure on $V$ which is integrable near each $\Sigma_{i}$ as in Lemma 3.1. Then, let $\left(\bar{V}, \bar{J}_{1}\right)$ be the almost complex manifold obtained by collapsing each $\Sigma_{i}$ to a point $z_{i}$. The collapsing map $\pi$ : $V \rightarrow \bar{V}$ is obviously holomorphic, and we just have to put a suitable symplectic form $\bar{\omega}$ on $\bar{V}$.

By [McD2, Lemma 2.1], we may choose the neighbourhood $N_{i}$ of $\Sigma_{i}$ so that its image $B_{i}=\pi\left(N_{i}\right)$ under $\pi$ has the complex analytic structure of the ball $B(\varepsilon)$ in $\mathbb{C}^{2}$ and so that $\omega\left|\partial N_{i}=\omega_{0}\right| \partial B\left(\lambda_{i}+\varepsilon\right)$, where $\omega\left(\Sigma_{i}\right)=\pi \lambda_{i}^{2}$. Therefore, we may define $\bar{\omega}$ to be the push-forward by $\pi$ of $\omega$ outside these balls $B_{i}=\pi\left(N_{i}\right)$, extended by suitable multiples of the standard form $\omega_{0}$ inside the $B_{i}$. Note that $\bar{\omega}$ does tame $\bar{J}_{1}$ since $\bar{J}_{1}$ equals $J_{0}$ in the ball $B_{i}$ and so is invariant under scalar multiplication.

(3.3) Proof of Theorem 1.1. We first show that the blowing down $(\bar{V}, \bar{\omega})$ constructed in Lemma 3.2 above is minimal. Suppose not, and let $\bar{\Sigma}$ be an exceptional curve in $\bar{V}$ in class $\bar{E}$. We can assume that $J_{1}$ and also $\bar{J}_{1}$ are $C^{\infty}$. Suppose that $\bar{J}_{1} \in \mathscr{U}(\bar{V})$. Then, by Lemma $3.1, \bar{E}$ has an embedded $\bar{J}_{1}$-holomorphic representative, $\bar{\Sigma}_{1}$ say. If this does not go through the points $z_{i}=\pi\left(\Sigma_{i}\right)$, it lifts to an exceptional curve in $V$ which is disjoint from the curves in $\mathscr{E}$, contradicting the maximality of $\mathscr{E}$. Even if it goes through some $z_{i}$, it can be easily moved to an embedded $\bar{J}$-holomorphic curve which does not meet any $z_{i}$ as follows.

Let $U=\bigsqcup_{i} B_{i}$. Because $\bar{J}_{1}$ is integrable inside $U$, we can move $\bar{\Sigma}_{1}$ inside $U$ so that it does not go through the $z_{i}$ (but remains $\bar{J}_{1}$-holomorphic in $U$ ) and then patch this new piece of curve to the old one somewhere outside $U$. This process (which is described in detail in [McD4, Lemma 4.3]) yields an embedded curve $\bar{\Sigma}$ which still lifts to a symplectically embedded curve in $V$ since it is $\bar{J}$-holomorphic for some $\bar{J}$ which equals $\bar{J}_{1}$ inside $U$.

Thus, it remains to deal with the case when the $\bar{J}_{1}$-holomorphic representative $\bar{\Sigma}_{1}$ is merely a cusp-curve. We can still move $\bar{\Sigma}_{1}$ away from the points $z_{i}$ as above, because there is a set $X \subset \bar{\Sigma}_{1}$ with a finite set of accumulation points such that $\bar{\Sigma}_{1}-X$ is embedded; see [McD1, Lemma 4.4(iii)]. There- 
fore, we may assume that $\bar{\Sigma}_{1}$ does not meet the $z_{i}$. In this case, there is a small neighbourhood $\overline{\mathscr{O}}$ of $\bar{J}_{1}$ in $\mathscr{J}(\bar{\omega})$ such that, for all $\bar{J} \in \overline{\mathscr{O}}$, the $\bar{J}$ holomorphic representative $\bar{\Sigma}_{J}$ of $\bar{E}$ is disjoint from all the $z_{i}$. Moreover, for the dense set of $\bar{J}$ in $\overline{\mathscr{O}} \cap \mathscr{U}_{\infty}$, Lemma 3.1 implies that $\bar{\Sigma}_{J}$ is embedded. Let $B$ be a neighbourhood of the set of points $\left\{z_{1}, \ldots, z_{k}\right\}$ which meets none of these $\bar{\Sigma}_{J}$. Then, because tameness is an open condition, if we choose $\bar{J}$ in $\overline{\mathscr{O}} \cap \mathscr{U}_{\infty}$ sufficiently close to $\bar{J}_{1}, \bar{J}$ lifts to an $\omega$-tame almost complex structure on $V-\pi^{-1}(B)$, which implies that the lift of $\bar{\Sigma}_{J}$ is symplectically embedded. Since this contradicts the maximality of the $\mathscr{E}$, the existence of the minimal manifold $(\bar{V}, \bar{\omega})$ is established.

It remains to consider its uniqueness. Observe first that the manifold $\bar{V}$ is uniquely determined by the choice of $\mathscr{E}$, and that the form $\bar{\omega}$ on $\bar{V}$ is also unique up to isotopy. We now claim that the symplectomorphism type of $(\bar{V}, \bar{\omega})$ is determined by the set $\left\{E_{1}, \ldots, E_{k}\right\}$ of homology classes of the curves in $\mathscr{E}$. To see this, note that, given $\left\{E_{1}, \ldots, E_{k}\right\}$, each corresponding set $\mathscr{E}$ of exceptional curves is $J$-holomorphic for some $\omega$-tame $J$. As in Lemma 3.1, we may suppose that $J \in \mathscr{U}_{\infty}$. Since $\mathscr{U}_{\infty}$ is path-connected, two homologous sets of curves $\mathscr{E}_{1}$ and $\mathscr{E}_{2}$, which are holomorphic with respect to $J_{1}$ and $J_{2}$ respectively, are isotopic through sets of $J_{t}$-holomorphic curves, where $J_{t}, 1 \leq t \leq 2$, is a path in $\mathscr{U}_{\infty}$ joining $J_{1}$ to $J_{2}$. Hence, there is a symplectic isotopy of $V$ which takes $\mathscr{E}_{1}$ to $\mathscr{E}_{2}$, and so the corresponding reduced manifolds $\left(\bar{V}_{1}, \bar{\omega}_{1}\right)$ and $\left(\bar{V}_{2}, \bar{\omega}_{2}\right)$ are symplectomorphic.

There is a relative version of minimality which may be stated as follows. If $\Lambda$ is a subgroup in $H_{2}(V ; \mathbb{Z})$, we say that $(V, \omega)$ is $\Lambda$-minimal if no element $E \in \Lambda$ may be represented by an exceptional curve. By choosing a maximal set $\mathscr{E}$ of disjoint exceptional curves which represent classes in $\Lambda$, one can easily prove

Theorem 3.4. Let $\Lambda$ be a subgroup in $H_{2}(V ; \mathbb{Z})$. Then there is a blowing down map $\pi_{\Lambda}:(V, \omega) \rightarrow(\bar{V}, \bar{\omega})$ such that $(\bar{V}, \bar{\omega})$ is $\bar{\Lambda}$-minimal, where $\bar{\Lambda}$ is the push-forward of $\Lambda$ by $\pi$.

If $\Lambda=\left\{A \in H_{2}(V ; \mathbb{Z}): A \cdot[C]=0\right\}$, this is Theorem 1.1(ii).

\section{SYMPLECTIC FIBRATIONS}

General remarks. We will say that a symplectic form $\omega$ on $V$ and a fibration $\pi: V \rightarrow M$ are compatible if $\omega$ is nondegenerate on the fibers of $\pi$. In this section we study such triples $(V, \omega, \pi)$, where $V$ is a 2-sphere bundle over a compact 2-manifold $M$. We will begin by giving a criterion for a form $\omega$ on $V$ to be compatible with some fibration.

Proposition 4.1 (see [G, 2.4.A]). Let $F$ be a symplectically embedded rational $B$-curve in a symplectic 4-manifold $(V, \omega)$ where $B$ is a simple homology class of self-intersection zero. Then there is a fibration $\pi: V \rightarrow M$ which is compatible with $\omega$ and has one fiber equal to $F$. 
Proof. Let $J^{\prime}$ be any $\omega$-tame almost complex structure which splits near $F$. (This means that a neighbourhood of $F$ is biholomorphic to a neighbourhood of $\{0\} \times S^{2}$ in the complex manifold $\mathbb{C} \times S^{2}$.) Then, if $f$ is a $J^{\prime}$-holomorphic parametrization of $F$, it follows from $(2.7)$ that $\left(f, J^{\prime}\right)$ is a regular point for the Fredholm projection operator $P_{B}$. Hence there is a nearby regular value $J$ for $P_{B}$ which admits a $J$-holomorphic $B$-curve $F_{1}$ which is close to $F$ and hence symplectically isotopic to $F$. Thus, by changing $J$ by a small symplectic isotopy (which does not affect the fact that it is regular) one may assume that $F$ itself is $J$-holomorphic.

Since $B$ is simple, (2.2) implies that the 2-dimensional manifold of unparametrized $J$-holomorphic $B$-curves is compact. Further, because $B \cdot B=0$, there is at most one $B$-curve through each point. It follows that the evaluation map

$$
e_{B}(J): M_{p}(J, B) \times_{G} S^{2} \rightarrow V
$$

given by $(f, z) \mapsto f(z)$ has degree 1 . Hence there is exactly one $J$-holomorphic $B$-curve through each point of $V$. Further, by (2.6), these curves will be embedded provided that $J$ is $C^{\infty}$. Thus these curves form the fibers of a continuous surjection $\pi: V \rightarrow M$ which is smooth by Lemma 3.5 of [McD2].

Note. In fact, when $B$ is simple, the $J$-holomorphic $B$-curves form the fibers of a smooth fibration $V \rightarrow M$ for every $C^{\infty}$ smooth element $J$ of $\mathscr{J}(\omega)$. For, since the set of regular elements is dense, the compactness theorem implies that there is a $J$-holomorphic $B$-curve through each point of $V$, and the proof that these are the fibers of a smooth fibration goes through as before.

This proposition applies whenever $V$ is a bundle over a Riemann surface $M$ of genus $>0$, since then the image of the Hurewicz homomorphism $\pi_{2}(V) \rightarrow$ $H_{2}(V)$ is generated by the class $B$ of the fiber, so that $B$ is always simple. However, if $V$ is a bundle over $S^{2}$ the class $B$ of the fiber need not be simple. We now show that $B$ is $J$-simple for almost all $J$. Let $\mathscr{U} \subset \mathscr{J}(\omega)$ be as defined in (2.4) with $K=\omega(B)$, and let $\mathscr{U}_{\infty}$ consist of all $C^{\infty}$-smooth elements of $\mathscr{U}$. Recall that $\mathscr{U}$ is open, dense, and path-connected.

Lemma 4.2. Let $(V, \omega)$ be a symplectic manifold which is diffeomorphic to an $S^{2}$-bundle over $S^{2}$ with fiber in class $B$, and suppose that $B$ is represented by a symplectically embedded curve $F$. Then $B$ is $J$-simple for all $J \in \mathscr{Q}^{\prime}$, and the conclusions of Proposition 4.1 hold for $F$.

Proof. Suppose first that $V=S^{2} \times S^{2}$ and let $A=\left[S^{2} \times \mathrm{pt}\right], B=\left[\mathrm{pt} \times S^{2}\right]$. If there is a $B$-cusp-curve, it must have one component $S_{i}$ with $c\left(S_{i}\right) \leq 0$ because $c(B)=2$ and $c$ takes only even values. Hence $B$ is $J$-simple as claimed. Therefore the proof of Proposition 4.1 remains valid, provided that we choose $J \in \mathscr{U}_{\infty}$. (Note that both $\mathscr{U}$ and $\mathscr{U}_{\infty}$ are invariant under the group of symplectic diffeomorphisms of $V$, so that it is permissible to change $J$ by a small symplectic isotopy in order to make $F_{1}$ coincide with $F$.) 
Next, suppose that $V$ is the total space of the nontrivial bundle. Then $V$ is diffeomorphic to $\mathbb{C} P^{2} \# \overline{\mathbb{C P}}^{2}$ and $H_{2}(V)$ has generators $L=\left[\mathbb{C} P^{1}\right]$ and $E$ (the class of the blown up point). Thus $L \cdot L=1, E \cdot E=-1$, and $B=L-E$. Since $c(L-E)=2$, an $(L-E)$-cusp-curve, whose components all have $c \geq 1$, must have exactly two components both with $c=1$. Thus one component is in class $B_{1}=k L-m E$ where $k, m \geq 1$ and $3 k-m=1$ (because $0<\omega\left(B_{1}\right)<\omega(B)$ and $\left.c\left(B_{1}\right)=1\right)$, and the other is in class $B_{2}=-(k-1) L+(m-1) E$. But then $B_{i} \cdot B_{i}<0$ for $i=1,2$ which implies that there is at most one such cuspcurve for each value of $k$. Further, because $(k L-m E) \cdot(\bar{k} L-\bar{m} E)<0$ when $k>m \geq 1$ and $\bar{k} \geq \bar{m} \geq 1, L-E$ is $J$-simple for each $J \in \mathscr{U}$ which admits $(L-E)$-curves, and if it is not simple there is at most one $J$-holomorphic $(L-E)$-cusp-curve.

Therefore, in order to prove the lemma it suffices to show that $(L-E)$-curves exist for all $J \in \mathscr{U}$. We do this by using some arguments from [McD2, Lemma 3.3]. By hypothesis, there is a symplectically embedded $(L-E)$-curve $F$, and we may choose a regular value $J$ for $P_{L-E}$ as before so that the evaluation map

$$
e_{L-E}(J): M_{p}(J, L-E) \times{ }_{G} S^{2} \rightarrow V
$$

has degree 1 . Given $J_{0} \in \mathscr{U}$, let $J_{t}, 0 \leq t \leq 1$, be a generic path in $\mathscr{U}$ from $J=J_{1}$ to $J_{0}$, and choose a generic path $x(t), 0 \leq t \leq 1$, in $V$ such that $x(t)$ does not lie on the $J_{t}$-holomorphic $(L-E)$-cusp-curve (if this exists). It is easy to see that the manifolds $e_{L-E}\left(J_{i}\right)^{-1}(x(i)), i=0,1$ are (compactly) cobordant: cf. Lemma 4.7. Thus, there is a $J_{0}$-holomorphic $(L-E)$-curve, as claimed.

Note. When $V=S^{2} \times S^{2}$ one can avoid the use of (2.6) in Proposition 4.1 by considering the intersections of the $B$-curves with the transverse family of curves in the class $A=\left[S^{2} \times \mathrm{pt}\right]$. See Lemma 4.6 below. Similar but more roundabout arguments are possible with $\mathbb{C} P^{2} \# \overline{\mathbb{C} P}^{2}$; see [McD2].

Definition 4.3. If $X$ is a closed subset of $V$, we will say that $\omega_{0}$ and $\omega_{1}$ are isotopic (rel $X$ ) if they may be joined by a family of cohomologous symplectic forms $\omega_{t}$ whose restrictions to $X$ are all equal. Further, they are isotopic (preserving $X$ ) if they may be joined by a family of cohomologous symplectic forms $\omega_{t}$ whose restrictions to $X$ are all nondegenerate.

Note that if $X$ is a submanifold of $V$ which is either $\omega_{0}$-symplectic or of codimension 1 (or more generally, if the kernel of $\omega_{0} \mid X$ has constant rank) then Moser's theorem implies that, given two forms $\omega_{0}$ and $\omega_{1}$ which are isotopic (rel $X$ ), there is a corresponding family of diffeomorphisms $g_{t}$ which are the identity on $X$ and are such that $g_{t}^{*}\left(\omega_{t}\right)=\omega_{0}$. Further, if the forms are isotopic (preserving $X$ ), we may choose the family $g_{t}$ so that $g_{t}(X)=X$. Similar remarks apply if $X$ is the union of two symplectic submanifolds, which intersect transversally in a symplectic submanifold. In particular, we can take $X$ to be the union of two transversally intersecting curves $\Gamma \cup F$. 
Lemma 4.4. Let $\pi: V \rightarrow M$ be an $S^{2}$-bundle as above, and let the forms $\omega_{0}$ and $\omega_{1}$ be compatible with $\pi$. Then the following conditions are equivalent:

(i) the forms are isotopic;

(ii) they are isotopic preserving $F$;

(iii) they may be joined by a family $\omega_{t}$ of cohomologous symplectic forms which are compatible with $\pi$.

Proof. Since the remarks above imply that (iii) $\Rightarrow$ (ii) $\Rightarrow$ (i), it suffices to prove that $(\mathrm{i}) \Rightarrow$ (iii). By hypothesis, there is an isotopy $\tau_{t}$ which joins $\omega_{0}$ to $\omega_{1}$. Let $i=0$ or 1 . Since $\pi$ is compatible with $\omega_{i}$, there is an $\omega_{i}$-tame $J_{i}$ such that the fibers of $\pi$ are $J_{i}$-holomorphic. We claim that we may suppose that $J_{i} \in \mathscr{U}_{\infty}\left(\omega_{i}\right)$. To see this, observe first that, as in Lemma 4.2, the class $[F]$ is $J_{i}$-simple. Hence, by the compactness theorem, it is $J$-simple for all $J$ near $J_{i}$. Therefore, each such $J$ defines a fibration $\pi_{J}$ of $V$. By choosing $J$ sufficiently close to $J_{i}$, we may suppose that there is an isotopy $g_{t}, 0 \leq t \leq 1$, such that $\pi_{J} \circ g_{1}=\pi$. Further, since compatibility with $\pi$ is an open condition, we may suppose that all the forms $g_{t}^{*}\left(\omega_{i}\right)$ are $\pi$-compatible. Therefore, we may replace $\omega_{i}$ by $\omega_{i}^{\prime}=g_{1}^{*}\left(\omega_{i}\right)$ and $J_{i}$ by the pull-back $J_{i}^{\prime}$ of $J$ by $g_{1}$. Observe that if we choose $J$ in $\mathscr{U}_{\infty}\left(\omega_{i}\right)$, then $J_{i}^{\prime} \in \mathscr{U}_{\infty}\left(\omega_{i}^{\prime}\right)$ as required.

Now, choose a path $J_{t} \in \mathscr{U}_{\infty}\left(\tau_{t}\right)$ joining $J_{0}$ to $J_{1}$ and let $\pi_{t}$ be the corresponding fibrations. Then $\pi_{1}=\pi_{0}=\pi$, and there is an isotopy $g_{t}$ such that $\pi_{t} \circ g_{t}=\pi_{0}=\pi$. Since the set of orientation preserving diffeomorphisms $g$ such that $\pi \circ g=\pi$ is path-connected, we may suppose that $g_{1}=\mathrm{id}$. Thus $g_{t}^{*}\left(\tau_{t}\right)=\omega_{t}$ is the desired path.

Note. Even though the $\omega_{t}$ above are compatible with $\pi$, there need not be a family of fiberwise diffeomorphisms $h_{t}$ (i.e., diffeomorphisms which project to diffeomorphisms of the base) such that $h_{t}^{*}\left(\omega_{t}\right)=\omega_{0}$. To see this, note that because compatibility is an open condition such $h_{t}$ would exist only if every closed form $\omega$ close to $\omega_{0}=d x \wedge d y+d u \wedge d v$ were the pull-back of $\omega_{0}$ under some diffeomorphism which has the form $(x, y, u, v) \mapsto\left(g^{1}, g^{2}, g^{3}, g^{4}\right)$, where $g^{3}$ and $g^{4}$ depend only on $u$ and $v$. However, if this were true $\omega-$ $d g^{3} \wedge d g^{4}=d g^{1} \wedge d g^{2}$ would have to contain all the terms in $\omega$ except that involving $d u \wedge d v$ and would always have to have zero square, which is clearly not always the case. Therefore, we will work with forms on $V$ which are compatible with $\pi$, under the relation of isotopy. In this connection it is worth pointing out that Guillemin and Lerman in [GL] define an invariant of the pair $(\omega, \pi)$ which is a closed 2-form $\omega_{\Gamma}$ on $V$ with the property that $\omega-\omega_{\Gamma}$ is the pull-back of some form on $M$. This invariant is called the coupling form, and exists whenever $\pi: V \rightarrow M$ is a fibration with simply-connected fibers which is compatible with a symplectic form on $V$. ( $V$ can have any dimension here.) Since $\omega_{\Gamma}$ depends only on $\omega$ and $\pi$, it transforms in an obvious way under fiberwise diffeomorphism, and provides a more sophisticated way of showing that a family $h_{t}$ such that $h_{t}^{*}\left(\omega_{t}\right)=\omega_{0}$ need not exist. 
Uniqueness of symplectic structures on $S^{2} \times S^{2}$. We now begin the study of the uniqueness question by looking at symplectic fibrations whose underlying bundle is the trivial bundle $\pi: S^{2} \times S^{2} \rightarrow S^{2}$, where $\pi$ is projection onto the first factor. Our results in the general case will follow by cutting and pasting. As always, $F$ will denote a chosen fiber of $\pi$. Further, we will say that a form $\omega$ is split on some subset $U$ of $V$ if we have chosen a diffeomorphism from $U$ to a product $U_{1} \times U_{2}$ and if this diffeomorphism takes $\omega$ to a form $\beta_{1} \oplus \beta_{2}$ where $\beta_{i}$ is the pull-back to $U$ of a symplectic form on $U_{i}$.

Proposition 4.5. If $\omega$ is a symplectic form on $S^{2} \times S^{2}$ which is compatible with $\pi$, then $\omega$ is isotopic to a split form.

Proof. We first show that there is a family $\omega_{t}, 0 \leq t \leq 1$, of (not necessarily cohomologous) symplectic forms on $S^{2} \times S^{2}$ such that:

(i) $\omega_{0}=\omega$;

(ii) each $\omega_{t}$ is compatible with $\pi$;

(iii) there is an $\omega_{1}$-symplectic section of $\pi$ in the class $A=\left[S^{2} \times \mathrm{pt}\right]$.

The desired result will then follow by standard arguments.

It is convenient to transfer the problem to $\mathbb{R}^{2} \times S^{2}$. To do this, first isotop $\omega \operatorname{rel} F$ to a form $\bar{\omega}$ which splits in a small neighborhood $N(F)$ of the fiber $F=\left\{z_{0}\right\} \times S^{2}$. Then, identify $\left(S^{2}-z_{0}\right) \times S^{2}$ with $\left(\operatorname{Int} I^{2}\right) \times S^{2}$ by a product diffeomorphism, where $I^{2}$ denotes the square $\left\{(x, y) \in \mathbb{R}^{2}: 0 \leq x, y \leq 1\right\}$. This takes $\bar{\omega}$ to a form $\tau$ which splits near $\left(\partial I^{2}\right) \times S^{2}$ and does not vanish on the fibers $\mathrm{pt} \times S^{2}$. Clearly, we may suppose that $\tau$ equals the standard split form $\tau_{0}=d x \wedge d y \oplus \sigma_{0}$ near $\left(\partial I^{2}\right) \times S^{2}$, where $\sigma_{0}$ is the standard area form on $S^{2}$, and may then extend it over $\mathbb{R}^{2} \times S^{2}$ by $\tau_{0}$.

We now show how to find a symplectic section $\Gamma$ of the fibration $\pi: \mathbb{R}^{2} \times$ $S^{2} \rightarrow \mathbb{R}^{2}$ which is constant (i.e., equal to $\mathbb{R}^{2} \times\left\{u_{0}\right\}$ ) outside some compact set. As Eliashberg pointed out in [E2], one can construct the section $\Gamma$ over the part of the space where $\tau$ is not split by considering the Hamiltonian flow $\psi_{t}$ of the function $x$. Observe that this is just the linear flow $y \mapsto y+t$ outside of $I^{2} \times S^{2}$, and that it preserves the hypersurfaces $x=$ const. On these hypersurfaces, it flows along the null direction of $\tau$ and so is everywhere transverse to the fibers of $\pi$ since these are symplectic.

Choose a point $u_{0}$ in $S^{2}$, and let $\gamma$ be the arc $\left\{\left(x, 0, u_{0}\right): 0 \leq x \leq 1\right\}$ in $\partial I^{2} \times S^{2}$. Then consider the surface $\Gamma=\bigcup_{t \geq 0}\left\{\psi_{t}(\gamma)\right\} \cap I^{2} \times S^{2}$. By construction $\Gamma$ is transverse to the fibers of $\pi$. Also, the restriction of $\tau$ to $\Gamma$ does not vanish since the initial arc $\gamma$ is transverse to the level sets of the Hamiltonian $x$. Note that $\Gamma$ coincides with the plane $u=u_{0}$ near the three faces $y=0$ and $x=0,1$, and has the form $(x, y, u=\lambda(x))$ over some strip $1-\varepsilon<y \leq 1$, where $\lambda$ is a loop in $S^{2}$ which is based at $u_{0}$.

We extend the section $\Gamma$ over the rest of $\mathbb{R}^{2}$ in two steps. First, let $\lambda_{t}(x)$, $0 \leq t \leq 1$, be a homotopy from the based loop $\lambda=\lambda_{0}$ to the constant loop 
$\lambda_{1}$, and then extend $\Gamma$ over the strip $\left\{(x, y) \times S^{2}: 0 \leq x \leq 1,1 \leq y \leq N\right\}$ by setting it equal to the graph of the map $(x, y) \mapsto \lambda_{\alpha(y)}(x)$ for a suitable function $\alpha$ which equals 0 near $y=1$ and 1 near $y=N$. If $N$ is sufficiently large, we may clearly choose $\alpha$ so that $\tau \mid \Gamma$ never vanishes. Further, we may choose the $\lambda_{t}$ so that the section $\Gamma$ thus constructed coincides with the constant section $\mathbb{R}^{2} \times\left\{u_{0}\right\}$ near the boundary $\partial$ of the rectangle $[0,1] \times[0, N]$ and is homologous $\left(\right.$ rel $\partial$ ) to this section. Finally extend $\Gamma$ over the rest of $\mathbb{R}^{2}$ by the constant section.

In order to transfer our results back to $S^{2} \times S^{2}$, choose a diffeomorphism $\theta: I^{2} \rightarrow[0,1] \times[0, N]$ which preserves the area form $d x \wedge d y$ near $\partial I^{2}$, and let $\tau_{1}$ be the pull-back of $\tau \mid[0,1] \times[0, N] \times S^{2}$ via $\theta$. Then $\tau_{1}$ admits a symplectic section $\Gamma_{1}$ which is constant near $\partial I^{2} \times S^{2}$. Since $\tau_{1}=\tau$ near $\partial I^{2} \times S^{2}, \tau_{1}$ gives rise to a symplectic form $\omega_{1}$ on $S^{2} \times S^{2}$ which clearly has the properties (ii) and (iii) above. Moreover, the path $\omega_{t}$ may be constructed in an obvious way from the restrictions of $\tau$ to the sets $[0,1] \times[0, s N] \times S^{2}$, $1 / N \leq s \leq 1$.

The proof is now completed by Lemmas 4.6 and 4.7 below, which are mild improvements of results in $[G, 2.4]$.

Lemma 4.6 (cf. $\left[\mathrm{G}, 2.4 . \mathrm{A}_{1}\right]$ ). Let $\omega$ be a symplectic form on $S^{2} \times S^{2}$ which is compatible with $\pi$ and suppose that there is a symplectically embedded section $\Gamma$ in class $A=\left[S^{2} \times \mathrm{pt}\right]$. Then $\omega$ is isotopic to a split form.

Proof. By Lemma 4.2 both classes $A$ and $B$ are $J$-simple for all $J \in \mathscr{U}$, and, by Proposition 4.1, for each $\omega$-tame $J \in \mathscr{U}$ there are two families of $J$-holomorphic curves, one in class $A$ and the other in class $B$. Since each $A$ curve meets each $B$-curve exactly once, it follows from (2.5) that these curves are all embedded. Thus, for each such $J$ we get a diffeomorphism $\pi_{B} \times \pi_{A}$ : $S^{2} \times S^{2} \rightarrow S^{2} \times S^{2}$ where the fibers of $\pi_{A}$ (resp. $\pi_{B}$ ) are the $J$-holomorphic $A$ - (resp. $B$-)curves. By arguing as in Proposition 4.1, we may choose $J \in \mathscr{U}$ so that $\Gamma$ and $F$ are $J$-holomorphic. Then, because $\pi_{B} \times \pi_{A}$ is determined by its restriction to $\Gamma \cup F$, we may suppose that it is the identity on $F$ and takes $\Gamma$ by any preferred diffeomorphism to $S^{2} \times\left\{u_{0}\right\}$ where $\left(z_{0}, u_{0}\right)=\Gamma \cap F$. Further, it is clear from the construction that $\pi_{B} \times \pi_{A}$ is isotopic to the identity map. Hence each split form $\omega_{0}$ is isotopic to its pull-back $\omega_{0}^{*}$ by $\pi_{B} \times \pi_{A}$, and it suffices to show that $\omega$ is isotopic to $\omega_{0}^{*}$, where $\omega_{0}$ is chosen to be homologous to $\omega$. To prove this, observe that $\omega_{0}^{*}$ is nondegenerate on the $J$-holomorphic $A$ - and $B$-curves. Further, these curves are $\omega_{0}^{*}$-orthogonal. It follows easily that $\omega_{0}^{*}$ tames $J$, so that the linear family $(1-t) \omega+t \omega_{0}^{*}, 0 \leq t \leq 1$, is an isotopy from $\omega$ to $\omega_{0}^{*}$.

Lemma 4.7. Suppose that $\omega_{t}, 0 \leq t \leq 1$, is a family of symplectic forms on $S^{2} \times S^{2}$ such that $\omega_{1}$ is split. Then $\omega_{0}$ is isotopic to a split form. 
Proof. This is obvious if the cohomology class $\left[\omega_{t}\right]$ is constant. Thus the interesting case is when $\left[\omega_{t}\right]$ varies.

Let $\mathscr{U}^{\prime}$ be $\bigcup_{t} \mathscr{U}_{t}$, where $t \in[0,1]$ and where $\mathscr{U}_{t}$ is the subset of $\mathscr{J}\left(\omega_{t}\right)$ defined in (2.4) with $K=\max _{t}\left\{\omega_{t}(A), \omega_{t}(B)\right\}$. Then $\mathscr{U}^{\prime}$ is path-connected. Further, if $J_{1}$ is the standard product almost complex structure on $S^{2} \times S^{2}$ (which, by hypothesis, is $\omega_{1}$-tame), then $J_{1} \in \mathscr{U}_{1}$ because the only $J_{1}$-holomorphic curves are graphs of holomorphic self-maps of $S^{2}$ and so lie in the classes $A+k B$ or $B+k A$ for $k \geq 0$. Choose some $C^{\infty}$-smooth element $J_{0} \in \mathscr{U}_{0}$ which is regular for $P_{A}$ and $P_{B}$, and let $\alpha(t), 0 \leq t \leq 1$, be a path in $\mathscr{U}^{\prime}$ joining $J_{0}$ to $J_{1}$. We may suppose that $\alpha$ is transverse to the operators $P_{A}^{\prime}$ and $P_{B}^{\prime}$, where $P_{A}^{\prime}$, for example, is the obvious projection of the manifold

$$
\mathscr{M}_{A}^{\prime}=\left\{(f, J) \in F \times \bigcup_{t} \mathscr{J}\left(\omega_{t}\right): f \text { is J-holomorphic }\right\}
$$

onto $\mathscr{J}^{\prime}=\bigcup_{t} \mathscr{J}\left(\omega_{t}\right)$. By Lemma 4.2, the class $A$ is $J_{t}$-simple for each $t$. Hence the set $P_{A}^{\prime-1}(\alpha) / G$ is a compact nonempty manifold which provides a cobordism between the manifolds $M\left(J_{0}, A\right)$ and $M\left(J_{1}, A\right)$ of $J_{0^{-}}$and $J_{1^{-}}$holomorphic $A$-curves. Further, the evaluation maps

$$
e_{A}\left(J_{i}\right): M_{p}\left(J_{i}, A\right) \times{ }_{G} S^{2} \rightarrow S^{2} \times S^{2}
$$

are (compactly) bordant. Since $e_{A}\left(J_{1}\right)$ has degree $1, e_{A}\left(J_{0}\right)$ must also have degree 1 , which implies, in particular, that $M_{p}\left(J_{0}, A\right)$ is nonempty. A similar argument shows that $M_{p}\left(J_{0}, B\right)$ is nonempty. Thus there are $J_{0}$-holomorphic $A$-and $B$-curves. Since these must be embedded by (2.6) (or (2.5)), the previous lemma implies that $\omega_{0}$ is isotopic to a split form.

This completes the proof of Proposition 4.5. Clearly this result is equivalent to the following.

Corollary 4.8. If $\omega$ is a symplectic form on $I^{2} \times S^{2}$ which is split near $\partial I^{2} \times S^{2}$, then $\omega$ is isotopic rel $\partial I^{2} \times S^{2}$ to a split form.

Uniqueness for the nontrivial $S^{2}$-bundle over $S^{2}$. Let us now consider the nontrivial $S^{2}$-bundle $\pi: X \rightarrow S^{2}$. We aim to show that, if $\omega$ is compatible with $\pi$, it is determined by its cohomology class up to isotopy and that its cohomology class $a$ satisfies the conditions in Theorem 1.3(i). If we identify $X$ with $\mathbb{C} P^{2} \# \overline{\mathbb{C} P}^{2}$ and use the basis $L, E$ of $H_{2}(X, \mathbb{Z})$ considered in Lemma 4.2, an easy calculation shows that these conditions are equivalent to requiring that both $a(F)=a(L-E)$ and $a(E)$ are positive. In [McD2, §4] we considered a closely related problem, looking at symplectic forms $\tau$ on $\mathbb{C} P^{2} \# \overline{\mathbb{C P}}^{2}$ which are nondegenerate on a fixed copy $S_{L}$ of the complex line $\mathbb{C} P^{1}$ in $X$. We proved that these forms are determined by their cohomology class $a$ up to a diffeomorphism which preserves $S_{L}$, and that $a(F)$ and $a(E)$ are positive. The second statement follows from an argument of Gromov's which shows that 
if this were not true, the class $L$ would be simple and the manifold $X$ would have to be $\mathbb{C} P^{2}$. To prove the first statement, we first showed that $\tau$ is symplectic on the fibers of some fibration $\pi_{\tau}: X \rightarrow S^{2}$, which is determined up to isotopy by the form $\tau$ and may be chosen so that $S_{L}$ is a section of $\pi_{L}$. Then, we used a cutting and pasting argument to show that any two cohomologous symplectic forms $\tau_{0}$ and $\tau_{1}$ which are nondegenerate on one fiber $F$ of $\pi_{\tau}$ and on the section $S_{L}$ are isotopic preserving $S_{L} \cup F$. Thus we showed

Lemma 4.9. Let $\omega_{0}$ and $\omega_{1}$ be cohomologous symplectic forms on $X$ which are nondegenerate both on $S_{L}$ and on one fiber $F$ of $\pi$. Then, there is an isotopy $\omega_{t}$ which preserves $S_{L} \cup F$ and joins $\omega_{0}$ to $\omega_{1}$. Further $\left[\omega_{0}\right]=\left[\omega_{1}\right]$ satisfies the conditions of Theorem 1.3(i).

Next, we prove the analogue of Lemma 4.7.

Lemma 4.10. Suppose that $\omega_{t}, 0 \leq t \leq 1$, is a family of symplectic forms on $X$ such that $\omega_{1}$ is nondegenerate on $S_{L} \cup F$. Then $\omega_{0}$ is isotopic to a form which is nondegenerate on $S_{L} \cup F$.

Proof. Let $J_{1}$ be the usual complex structure on $X=\mathbb{C} P^{2} \# \overline{\mathbb{C P}}^{2}$. We may suppose that $\pi$ is a holomorphic map from $\left(X, J_{1}\right)$ to the Riemann sphere, and that $S_{L}$ is a $J_{1}$-holomorphic copy of $\mathbb{C} P^{1}$. By Lemma 4.9 , we may suppose that $\omega_{1}$ is a Kähler form with respect to $J_{1}$. Then $J_{1} \in \mathscr{U}\left(\omega_{1}\right)$ and is regular for the classes $L, L-E$, and $E$ (with notation as in Lemma 4.2).

Define $\mathscr{U}^{\prime}$ as in Lemma 4.7, and choose a path $\alpha(t) \in \mathscr{U}^{\prime}, 0 \leq t \leq 1$, which begins at a generic element of $\mathscr{U}_{0}$ and ends at $J_{1}$, and is transverse to the projection operators $P_{A}^{\prime}$ for $A=L, L-E$, and $E$. Observe that, if $a \in H^{2}(X)$ is such that $a^{2} \neq 0$, then $a(L-E) \neq 0$. Hence, because $\omega_{1}(L-E)>0$, we must have $\omega_{t}(L-E)>0$ for all $t$. Further, by combining the arguments of Lemma 4.2 with those of Lemma 4.7, one easily sees that, for all $t, L-E$ is a $J_{t}$-simple class which has $J_{t}$-holomorphic representatives.

We next show that there is a $J_{0}$-holomorphic $L$-curve $\bar{S}_{L}$. We use essentially the same argument as before, but now must cope with the fact that $L$ is no longer a simple class. However, because $(k L-m E) \cdot(L-E)=k-m$, the only possible $J_{t}$-holomorphic $L$-cusp-curves have type $(L-E, E)$. Let $X_{t}$ be the set of pairs $(x, y) \in V \times V$ such that $x$ and $y$ lie on the same $J_{t^{-}}$ holomorphic $L$-cusp-curve. Thus $(x, y) \in X_{t}$ if either of $x$ and $y$ lie on the unique $J_{t}$-holomorphic $E$-curve or if they both lie on the same $J_{t}$-holomorphic $(L-E)$-curve. Since $X_{t}$ has codimension $\geq 2$, one can choose a path $\beta(t)=$ $\left(x_{t}, y_{t}\right) \in V \times V-X_{t}$ for $0 \leq t \leq 1$ such that $x_{1}$ and $y_{1}$ are distinct points on $S_{L}$. Now, as in the proof of Lemma 4.7, consider the evaluation map

$$
e_{L}(\alpha): \bigcup_{t} M_{p}\left(J_{t}, L\right) \times_{G}\left(S^{2} \times S^{2}\right) \rightarrow V \times V .
$$

We may jiggle $\beta$ so that it is transverse to $e_{L}(\alpha)$. Then, because $\beta(t)$ avoids the $L$-cusp-curves, the manifold $\bigcup_{t} e_{L}(\alpha)^{-1}(\beta(t))$ is compact, and its boundary 
lies over $\beta(0) \cup \beta(1)$. Since, by construction, it has exactly one point which lies over $\beta(1)$, it must have at least one point lying over $\beta(0)$. In other words, there must be a $J_{0}$-holomorphic $L$-curve $\bar{S}_{L}$, as claimed. Note that it is embedded by $(2.5)$.

Thus, there is a $J_{0}$-holomorphic $L$-curve $\bar{S}_{L}$ and a $J_{0}$-holomorphic $(L-E)$ curve $\bar{F}$. It follows easily from their construction that they are isotopic to $S_{L}$ and $F$, respectively. Since $\omega_{0}$ is nondegenerate on $\bar{S}_{L}$ and $\bar{F}$, the result follows.

Proposition 4.11. Suppose that $\omega$ is a symplectic form on $X$ which is compatible with $\pi$. Then $\omega$ satisfies the conditions of Theorem 1.3(i) and is determined by its cohomology class up to isotopy.

Proof. Let $F$ be one of the fibers of $\pi$. Then, by the symplectic neighbourhood theorem, we may identify a neighbourhood $\pi^{-1}(U)$ of $F$ with $U \times S^{2}$ in such a way that $\omega$ corresponds to a split form. Therefore, we may cut out a small piece $\left(Y_{1}, \tau_{1}\right)$ from $X$, in such a way that $\tau_{1}$ is the restriction of the standard split form $\tau_{0}=d x \wedge d y \oplus \sigma_{0}$ to $Y_{1}=[0,1] \times\left[0, T_{1}\right] \times S^{2} \subset \mathbb{R}^{2} \times S^{2}$, and $\pi$ corresponds to the obvious projection of $Y_{1}$ onto $[0,1] \times\left[0, T_{1}\right]$. As explained in [McD2, (4.4)], the complementary piece $\left(Y_{2}, \tau_{2}\right)$ may be described as follows. Let $\mu: S^{2} \mapsto[0, a]$ be the moment map of the standard action $(\theta, z) \mapsto \theta \cdot z$ of the circle on $S^{2}$ by rotation, and let $f$ be a nondecreasing function from $[0,1]$ onto itself which is constant near the endpoints. Then $Y_{2}$ is the set

$$
Y_{2}=\left\{(x, y, z): 0 \leq x \leq 1,-f^{\prime}(x) \mu(z) \leq y \leq 1, z \in S^{2}\right\} \subset \mathbb{R}^{2} \times S^{2},
$$

and the form $\tau_{2}$ equals $\tau_{0}$ near $\partial Y_{2}$. To see this, consider the map $\alpha$ which takes the face $\left\{y=T_{1}\right\}$ of $Y_{1}$ to the face $\left\{y=-f^{\prime}(x) \mu(z)\right\}$ of $Y_{2}$ and is defined by

$$
\alpha(x, y, z)=\left(x, y-T_{1}-f^{\prime}(x) \mu(z), f(x) \cdot z\right) .
$$

It is not hard to check that $\alpha^{*}\left(\tau_{0}\right)=\tau_{1}$ so that $Y_{1} \cup_{\alpha} Y_{2}$ has a symplectic form $\tau$ which is split near its boundary. Hence, if we identify this boundary to a single fiber, we get a symplectic manifold $(X, \omega)$ which fibers over $S^{2}$. Note that this fibration $X \rightarrow S^{2}$ is nontrivial because of the twist in the gluing map $\alpha$.

Under this identification the map $\pi$ gives rise to a fibration $\pi_{2}: Y_{2} \rightarrow I^{2}$ which is defined on $\partial Y_{2}$ by

$$
\begin{aligned}
\pi_{2}(x, y, z) & =(x, y) \quad \text { if } y>0, \\
& =(x, 0) \quad \text { if } y \leq 0 .
\end{aligned}
$$

Since we can freely choose the identification of $\operatorname{Int}\left(X-Y_{1}\right)$ with $\operatorname{Int} Y_{2}$, we will suppose, as we may, that $\pi_{2}$ has the form $(x, y, z) \mapsto(x, g(x, y, z))$, and that the surfaces $\Gamma_{i}=\mathbb{R}^{2} \times\left\{z_{i}\right\} \cap Y_{2}, i=0,1$, are transverse to the fibers of $\pi_{2}$, where $z_{0}$ and $z_{1}$ are the fixed points of the circle action on $S^{2}$, named 
so that $\mu\left(z_{0}\right)=0$ and $\mu\left(z_{1}\right)=a$. (It is easy to see that the section of $X$ corresponding to $\Gamma_{1}$ is isotopic to $S_{L}$ and that $\Gamma_{0}$ corresponds to a curve in class $E$.)

As in the proof of Proposition 4.4, we now construct a family $\bar{\omega}_{t}, 0 \leq t \leq 1$, of symplectic forms on $Y_{2}$ such that:

(i) $\bar{\omega}_{t}=\tau_{2}$ near $\partial Y_{2}$ for all $t$ and on $Y_{2}$ when $t=0$;

(ii) each $\bar{\omega}_{t}$ is nondegenerate on the fibers of $\pi_{2}$;

(iii) there is a section $\Gamma$ of $\pi_{2}$ which coincides with $\Gamma_{1}$ near $\partial Y_{2}$, is homologous to $\Gamma_{1}\left(\operatorname{rel} \partial Y_{2}\right)$, and is symplectic with respect to $\omega_{1}$.

To do this, one starts with the arc $\gamma(t)=\left(t,-f^{\prime}(t) a, z_{1}\right)$ (where $\left.a=\mu\left(z_{1}\right)\right)$, and constructs a section over $Y_{2} \cup[0,1] \times[1, N] \times S^{2}$ using the Hamiltonian flow of $x$. Note that, by our choice of $\pi_{2}$, the level sets of the function $x$ are unions of fibers of $\pi_{2}$, so that the argument goes through just as before.

It follows easily that the original form $\omega=\omega_{0}$ on $X$ is part of a family $\omega_{t}$ which satisfies the hypotheses of Lemma 4.10. Hence the desired result follows from Lemmas 4.10 and 4.9.

Since all the isotopies considered above are constant near the fiber $F$ of $\pi$, we have shown

Corollary 4.12. If $\tau$ is any symplectic form on $Y_{2}$ which equals the standard split form $\tau_{0}$ near $\partial Y_{2}$, then $\tau$ is isotopic to $\tau_{0} \operatorname{rel} \partial Y_{2}$.

Bundles over surfaces of higher genus. Now let us consider a symplectic $S^{2}$ bundle $\pi: V \rightarrow M$ over a Riemann surface $M$ of genus $g>0$. First of all, we describe the "standard forms" on $V$. If $V$ is trivial, these are just the split forms. Otherwise, we identify $V$ with the projectivization $P\left(L_{1} \oplus \mathbb{C}\right)$ of a rank 2 holomorphic bundle $L_{1} \oplus \mathbb{C}$ over $M$, where the line bundle $L_{1}$ has Chern number 1 . Then the associated complex structure $J$ is invariant under the action of $S^{1}$ induced by multiplication by $e^{2 \psi i t}$ in $L_{1}$, and $P\left(L_{1} \oplus \mathbb{C}\right)$ has invariant Kähler forms which are nondegenerate on the fibers of $\pi$ and on the two fixed point sets, $M_{+}$(the zero section of $L_{1}$ ) and $M_{-}$(the zero section of $\mathbb{C}$ ). See [Au]. If $\left\{b_{+}, b_{-}\right\}$is the dual basis of $H^{2}(V)$, it is easy to check that $b_{+}^{2}(V)=-b_{-}^{2}(V)=1$ and that $\left[M_{+}\right]-\left[M_{-}\right]$is the class $[F]$ of the fiber. An easy calculation now shows that $a=\lambda_{+} b_{+}+\lambda_{-} b_{-}$is the class of one of these Kähler forms if and only if $\lambda_{+}>\lambda_{-}>0$, and hence if and only if the conditions in Theorem 1.3(i) hold.

We aim to show that any $\pi$-compatible symplectic form $\omega$ on $V$ is isotopic to one of the above "standard" forms. We will suppose that $M$ has been assembled from a $4 g$-sided polygon $P \subset \mathbb{R}^{2}$ by identifying its sides in the standard way $a_{1} a_{2} a_{1}^{-1} a_{2}^{-1} a_{3} a_{4} \cdots a_{2 g}^{-1}$. The vertices of $P$ are identified to a point $x_{0}$ in $M$ and the sides form embedded loops $\lambda_{1}, \ldots, \lambda_{2 g}$ which intersect only at $x_{0}$. Let $\Lambda=\lambda_{1} \cup \cdots \cup \lambda_{2 g}$ and let $F$ be the fiber at $x_{0}$. If $\pi$ is trivial, we identify $\left(V, \pi^{-1}(\Lambda)\right)$ with $\left(M \times S^{2}, \Lambda \times S^{2}\right)$ in the obvious way, and if $\pi$ 
is not trivial, we choose some identification of $\pi^{-1}(\Lambda)$ with $\Lambda \times S^{2}$. It then makes sense to talk about a form being split near $\pi^{-1}(\Lambda)$.

Lemma 4.13. Any $\pi$-compatible symplectic form which splits near $\pi^{-1}(\Lambda)$ is isotopic to a standard form.

Proof. When $\pi$ is the trivial bundle this follows immediately from Corollary 4.8. For, one can cut $V$ along $\pi^{-1}(\Lambda)$ to obtain a form $\tau$ on $P \times S^{2}$ which is split near the boundary. If $\pi$ is nontrivial use Corollary 4.12 instead.

Lemma 4.14. If $\omega$ is compatible with $\pi$, there is a family $\omega_{t}$ of symplectic forms on $V$ with $\omega_{0}=\omega$ such that $\omega_{1}$ splits near $\pi^{-1}(\Lambda) \cong \Lambda \times S^{2}$.

Proof. Let $D$ be a small neighbourhood of $x_{0}$ in $M$. By the symplectic neighbourhood theorem, we may suppose that $\omega$ splits on $\pi^{-1}(D) \cong D \times S^{2}$. The family $\omega_{t}$ will consist of forms which equal $\omega$ in $V-\pi^{-1}(D)$ and which equal $\omega+\pi^{*}\left(\rho_{t} d x \wedge d y\right)$ in $\pi^{-1}(D)$, where $\rho_{t}$ is a function on $D$ which is zero on the boundary and is $\geq 0$ inside.

Let $D_{0}$ be a smaller neighbourhood of $x_{0}$ inside $\operatorname{Int} D$. For each $i$, choose a diffeomorphism of a neighbourhood of $\pi^{-1}\left(\lambda_{i}\right)$ in $\pi^{-1}\left(M-D_{0}\right)$ with the set $W=[0,1] \times[-\varepsilon, \varepsilon] \times S^{2}$ which extends the above diffeomorphism $\pi^{-1}(D) \rightarrow$ $D \times S^{2}$, and is such that $\omega$ is nondegenerate on each fiber of the projection $\pi: W \rightarrow[0,1] \times[-\varepsilon, \varepsilon]$. We will show that, for suitable $\rho_{t}$, there is an isotopy $\Psi_{1}$ with support in Int $W$ such that $\Psi_{1}^{*}\left(\omega_{1}\right)$ splits on $H=[0,1] \times\{0\} \times S^{2}$. By the symplectic neighbourhood theorem, $\Psi_{1}^{*}\left(\omega_{1}\right)$ must then be isotopic to a form which splits near $H$, and, because we can do this for each $i$ separately, the lemma follows.

As a first attempt at finding $\Psi_{1}$, let $\xi$ be a vector field on $H$ which points along the null-directions of $\omega \mid H$. Since $\omega$ is nondegenerate on the fibers of $\pi, \xi$ is transverse to these fibers and we can normalize it so that it covers the vector field $\partial / \partial s$ on $[0,1]$. Then $\xi=\partial / \partial s$ near $\partial H$, so that its flow has the form $(s, z) \mapsto\left(s+t, g_{t}(s, z)\right)$, and the diffeomorphism $h_{s}$ of $S^{2}$, defined by $h_{s}(z)=g_{s}(0, z)$, equals the identity for $s$ near 0 and is independent of $s$ for $s$ in some interval $[1-\nu, 1]$. Define $G: H \rightarrow H$ by setting $G(s, z)=\left(s, h_{s}(z)\right)$. It is easy to check that $G^{*}(\omega)$ splits on $H$. Unfortunately, this $G$ does not quite do for $\Psi_{1}$ since it may not equal the identity on the boundary component $\{1\} \times S^{2}$ of $H$.

To remedy this, we alter $G$ on $[1-\nu, 1] \times S^{2}$ to an embedding $G^{\prime}$ of this set into $[1-\nu, 1] \times[-\varepsilon, \varepsilon] \times S^{2} \subset W$ which equals $G$ near $\{1-\nu\} \times S^{2}$, is the identity near $\{1\} \times S^{2}$, and which pulls back $\omega$ to a split form. Note that we can choose $\nu$ so small that $[1-\nu, 1] \times[-\varepsilon, \varepsilon] \subset D-D_{0}$. We will choose $\rho_{t}$ so that $\omega_{t}=\omega$ in $[0,1-\nu] \times[-\varepsilon, \varepsilon] \times S^{2}$, and so that $([1-\nu, 1] \times[-\varepsilon, \varepsilon] \times$ $\left.S^{2}, \omega_{t}\right)$ is symplectomorphic to a subset of the form $\{(x, y, z):|y| \leq \beta(x)\}$ in $\left([1-\nu, 1] \times \mathbb{R} \times S^{2}, \tau_{0}\right)$. Clearly, we may choose $\rho_{t}$ so that $\beta$ is any 
function which is $\geq \varepsilon$, with equality when $x$ is near $1-\nu$ and 1 . Now define $G^{\prime}$ by the formula

$$
(s, z) \mapsto\left(s,-f_{s}\left(h_{1}(z)\right), h_{\alpha(s)}(z)\right)
$$

where the values of $\alpha(s)$ decrease from 1 to 0 , and where $f_{s}: S^{2} \rightarrow \mathbb{R}$ is a (time-dependent) Hamiltonian which generates the $\sigma_{0}$-preserving flow $h_{\alpha(s)} h_{1}^{-1}$ on $S^{2}$, and is zero for $s$ near $1-\nu$ and 1 . (Since each $f_{s}$ must be constant for $s$ near $1-\nu$ and 1 , we can adjust it by a function of $s$ to make it zero there.) It is easy to check that $G^{\prime *}(\tau)|H=\tau| H$ as required.

Lemma 4.15. If $\omega$ is nondegenerate on some section $\Gamma$ of $\pi$, we may choose the family $\omega_{t}$ of Lemma 4.14 so that its cohomology class is constant and so that $\omega_{t}|\Gamma=\omega| \Gamma$ for all $t$.

Proof. First observe that we may identify $\pi^{-1}(\Lambda \cup D)$ with $(\Lambda \cup D) \times S^{2}$ in such a way that $\Gamma \cap \pi^{-1}(\Lambda \cup D)$ is taken to $(\Lambda \cup D) \times\left\{z_{0}\right\}$ for some point $z_{0} \in S^{2}$. Further, we may isotop $\omega$ near $(\Lambda \cup D) \times\left\{z_{0}\right\}$ so that $\omega$ splits near $(\Lambda \cup D) \times\left\{z_{0}\right\}$ as well as in $\pi^{-1}(D)$. Hence, we may choose the identification of $W$ with a neighbourhood of $\pi^{-1}\left(\lambda_{i}\right)$ in such a way that $\omega$ splits near $[0,1] \times\{0\} \times\left\{z_{0}\right\}$. Then the diffeomorphisms $g_{s}$ will be the identity in a neighbourhood $U$ of $\left\{z_{0}\right\}$, and we may assume that $G^{\prime}=G$ there. It follows that we may choose $\Psi_{1}$ to be the identity on $\Gamma$. Thus, the family $\omega_{t}$ as constructed in Lemma 4.14 consists of forms which are nondegenerate on $\Gamma$.

We now change $\omega_{t}$ to $\bar{\omega}_{t}=\lambda_{t}\left(\omega_{t}+\mu_{t} \rho\right)$ where $\rho$ is a closed 2-form on $V$ whose integral over $\Gamma$ (resp. $F$ ) is 0 (resp. 1) and where the numbers $\lambda_{t}$ and $\mu_{t}$ are chosen so that $\left[\bar{\omega}_{t}\right]$ is independent of $t$. If $V$ is the product $M \times S^{2}$ and $\Gamma=M \times \mathrm{pt}$, we may choose $\rho$ to the pull-back of a form on the fiber $S^{2}$ with support in $U$. In general, we identify a neighbourhood $N(\Gamma)$ of $\Gamma$ with an $S^{1}$-invariant neighbourhood of the zero section in a complex line bundle over $\Gamma$, and let $\rho$ be the pull back of an $S^{1}$-invariant form on $\mathbb{C}$ with support near zero. Thus $\rho$ restricts to 0 on $\Gamma$, and restricts on $H=[0,1] \times S^{2} \subset W$ to a form which is pulled back from $S^{2}$. It follows that the kernel of $\rho \mid H$ agrees with that of $\omega_{t}$ near the line $[0,1] \times\left\{z_{0}\right\}$ in $H$, so that the embeddings $G$ and $G^{\prime}$ as constructed in Lemma 4.14 are symplectic with respect to $\bar{\omega}_{t}$, as required.

Lemma 4.16. Suppose that the $\pi$-compatible form $\omega_{0}$ may be joined to a standard form $\omega_{1}$ by a family $\omega_{t}, 0 \leq t \leq 1$, of symplectic forms on $V$. Then $\omega_{0}$ is nondegenerate on a section of $\pi$.

Proof. Suppose first that $V$ is the nontrivial bundle over $M$ where genus $M=$ $g>1$, and let $D=\left[M_{+}\right]+(g-1)[F]$, where $\left[M_{+}\right]$is the section with selfintersection +1 as described above. Then $D \cdot D=2 g-1$, which, by Lemma 2.8 , is the smallest possible value for regular curves. Define $\mathscr{U}^{\prime}=\mathscr{U}^{\prime}(K, g)$ as in Lemma 4.7 (with $K=\max _{t} \omega_{t}(D)$ ), and let $J_{0}$ be a generic element of $\mathscr{U}_{0}$. 
We claim that the lemma will follow if we show that there is a $J_{0}$-holomorphic $D$-curve $C_{0}$. For, because $B \cdot D=1$, such a curve $C_{0}$ must intersect each $J_{0}$-holomorphic $B$-curve exactly once, and so be a section of the fibration $\pi_{0}$ which is defined by these curves. To get a section of $\pi$, we may take a limit of the curves $C_{0}$ as $J_{0}$ converges to an almost complex structure $J_{\pi}$ whose corresponding fibration is $\pi$. If this limit is a curve, we are done. But, if it is a cusp-curve, it must be the union of a number of fibers of $\pi$ together with some section $\Gamma_{0}$ of $\pi$. Thus, in either case, there is an $\omega_{0}$-symplectic section of $\pi$.

One shows that $C_{0}$ exists much as in Lemma 4.10. First of all, we must establish the existence of an $\omega_{1}$-symplectic section which belongs to a wellunderstood family of holomorphic curves. We do this as follows. Because the space of standard forms on $V$ is connected we may suppose that

$$
\omega_{1}\left(\left[M_{+}\right]-g[F]\right)>0,
$$

i.e., that $\left[\omega_{+}\right]=\lambda_{+} b_{+}+\lambda_{-} b_{-}$where $g \lambda_{+}>(g-1) \lambda_{-}$. Then $\omega_{1}$ tames not only the "standard" complex structures described above coming from the identification of $V$ with $P\left(L_{1} \oplus \mathbb{C}\right)$, but also those coming from a properly chosen identification of $V$ with $P\left(L_{2 g-1} \oplus \mathbb{C}\right)$, where $L_{2 g-1}$ has Chern number $2 g-1$. (This holds because $\omega_{1}$ has positive integral on the exceptional divisor $M_{-}=P\left(L_{2 g-1} \oplus 0\right)$. See Lemma 3.1 of [McD1] for a similar argument.) Let $J_{1}^{\prime}$ be a complex structure on $V$ coming from an identification of $V$ with $P\left(L_{2 g-1} \oplus \mathbb{C}\right)$, and let $\Gamma$ be the $J_{1}^{\prime}$-holomorphic section $P(0 \oplus \mathbb{C})$ which has class $D$. By Lemma $2.8, \Gamma$ has a parametrization $f_{1}:\left(\Sigma_{g}, \bar{J}\right) \rightarrow\left(V, J_{1}^{\prime}\right)$ such that $\left(f_{1}, \bar{J}, J_{1}^{\prime}\right)$ is a regular point for $P_{D}$.

We claim that there is an open set $U \subset V \times \cdots \times V$ such that the evaluation map

$$
e=e_{D, g}\left(J_{1}^{\prime}\right): M_{p}\left(J_{1}^{\prime}, \mathscr{T}_{g}, D\right) \times S^{2} \times \cdots \times S^{2} \rightarrow V \times \cdots \times V
$$

given by $\left(f, z_{1}, \ldots, z_{g}\right) \mapsto\left(f\left(z_{1}\right), \ldots, f\left(z_{g}\right)\right)$, maps $e^{-1}(U)$ bijectively onto $U$. To see this, observe first that, by the index formula of (2.1), both sides have the same dimension. Next observe that, because $D \cdot\left[M_{-}\right]=0$, every $D$-curve sits inside $V-M_{-} \equiv L_{2 g-1}$. In fact, the $D$-curves are just the $J_{1}^{\prime}$-holomorphic sections of $L_{2 g-1}$. By Riemann-Roch, the $\mathbb{C}$-dimension $h^{0}\left(\Sigma_{g}, L_{2 g-1}\right)$ of the space of holomorphic sections of $L_{2 g-1}$ is $g$. Further, it follows from the properties of linear systems (see, e.g., [GH, Chapter 2, §3]) that $\left(f_{1}, z_{1}, \ldots, z_{g}\right)$ is a regular point of $e$ for generic $\left(z_{1}, \ldots, z_{g}\right)$. Thus $e$ maps some neighbourhood of $\left(f_{1}, z_{1}, \ldots, z_{g}\right)$ diffeomorphically onto some neighbourhood $U$ of $\underline{w}=e\left(f_{1}, z_{1}, \ldots, z_{g}\right)$. But, for any $\underline{x} \in U, e^{-1}(\underline{x})$ cannot contain more than one point, for if it did, the linearity of the space of sections of $L_{2 g-1}$ would imply that $e^{-1}(\underline{x})$ is a subspace and so could contain no regular points. This proves the claim.

As in the proof of Proposition 4.1, there is a regular value $J_{1}$ for the operator $P_{D}$ which belongs to the set $\mathscr{U}_{\infty}(K, g)$ of $(2.4)$ (where $\left.K=\omega(\Gamma)\right)$ and is such 
that $\Gamma$ has a $J_{1}$-holomorphic parametrization $f$. (Note that $J_{1}^{\prime}$ does not belong to $\mathscr{U}_{\infty}(K, g)$ since $c\left(M_{-}\right)<1-g$.) Further, we may choose $J_{1}$ to be so close to $J_{1}^{\prime}$ that, for some open subset $U_{1} \subset U$, the evaluation map $e_{1}=e_{D, g}\left(J_{1}\right)$ maps $e_{1}^{-1}\left(U_{1}\right)$ bijectively onto $U_{1}$. Thus, one might say that $e_{1}$ has degree 1 over the points of $U_{1}$. Let $J_{t}$ be a generic family of $\omega_{t}$-tame almost complex structures in $\mathscr{U}^{\prime}$ which join $J_{1}$ to the element $J_{0}$ considered in the first paragraph of this proof, and let $\beta(t)$ be a path in $V \times \cdots \times V$ which ends at $\beta(1) \in U_{1}$. We may suppose that $\beta(t)$ is generic, so that it is transverse to $e_{D, g}\left(J_{1}\right)$ (as well as to any other evaluation map under consideration). The arguments of Lemma 4.10 will show that there is a $J_{0}$-holomorphic $D$-curve, provided that the path $\beta(t)$ may be chosen so that $\beta(t)$ does not lie on the image of any $J_{t}$-holomorphic $D$-cusp-curve. Therefore we must analyse the $J$-holomorphic $D$-cusp-curves, where $J \in \mathscr{U}_{t}$ for some $t$.

As mentioned above, any such cusp-curve must be the union of a number of spheres which represent the class $[F]$ of the fiber, together with some $J$ holomorphic curve $C_{m}$ which represents a class $D-m[F]$ where $m>0$. We will say that such a cusp-curve has type $m$. Since $C_{m}$ is a section of the fibration $\pi_{J}$, it is an embedded curve of genus $g$. Hence, because $c\left(C_{m}\right)=1-2 m>-g$ by definition of $\mathscr{U}_{\infty}(K, g), m<(g+1) / 2$. Taking $p$ to be the largest of these values of $m$, we find that the class $\left[C_{p}\right]$ is $J$-simple, and that the image of $e_{[C p], g}(J)$ is a compact set of codimension $\geq 4 p$. Since a $D$-cusp-curve is the union of such a curve $C_{p}$ with $p$ fibers, the set of $g$-tuples in $V \times \cdots \times V$ which lie on a $D$-cusp-curve of type $p$ has codimension $\geq 2 p$. Therefore, we may choose $\beta(t)$ to avoid these cusp-curves. Now consider cusp-curves of type $p-1$. Their image under $e_{[C p+1], g}(J)$ is a noncompact set of codimension $\geq 2(p-1)$ which accumulates on the image of the set of $D$-cusp-curves of type $p$. Hence, this image intersects a neighbourhood of $\beta$ in a compact set, so that we may arrange that $\beta$ avoids them. Continuing in this way, we see that we can suppose that $\beta$ avoids all $D$-cusp-curves, as required.

This completes the proof in the case when $g>1$ and $V$ is nontrivial. The other cases are essentially the same. When $g=1$ one has to remember the reparametrization group $T^{2}$ of the torus $\Sigma_{1}$, and when $V$ is trivial one should take $D$ so that $D \cdot D=2 g$.

Proposition 4.17. Let $\pi: V \rightarrow M$ be an $S^{2}$-bundle over a Riemann surface $M$. Then any two cohomologous $\pi$-compatible symplectic forms are isotopic and satisfy the conditions of Theorem 1.3(i) .

Proof. We claim that every $\pi$-compatible symplectic form $\omega$ is isotopic to a $\pi$-compatible symplectic form which splits near $\pi^{-1}(\Lambda)$. The result then follows from Lemma 4.13. To prove the claim, first use Lemma 4.14 to find a family of $\pi$-compatible symplectic forms $\omega_{t}$ with $\omega_{0}=\omega$ such that $\omega_{1}$ splits near $\pi^{-1}(\Lambda)$. Then, by either Corollary 4.8 or Corollary 4.12, $\omega_{1}$ is isotopic to a standard form $\omega_{1}^{\prime}$. Lemma 4.16 now implies that $\omega_{0}$ admits 
a symplectic section, and so, by Lemma $4.15, \omega=\omega_{0}$ is isotopic to a $\pi$ compatible symplectic form which splits near $\pi^{-1}(\Lambda)$.

Propositions 4.5, 4.11, and 4.17 clearly constitute a proof of Theorem 1.3.

\section{Uniqueness of symplectic sections.}

Proposition 4.18. Let $\pi: V \rightarrow M$ be an $S^{2}$-bundle over a Riemann surface of genus $g \geq 0$, and let $\omega$ be a compatible form on $V$, which is nondegenerate on two homologous sections $\Gamma_{0}$ and $\Gamma_{1}$ such that $\Gamma_{i} \cdot \Gamma_{i} \geq 2 g-1$. Then, there is a symplectic isotopy $g_{t}$ which preserves one fiber $F$ of $\pi$ and is such that $g_{1}\left(\Gamma_{0}\right)=\Gamma_{1}$.

Note. It follows easily from Moser's theorem that this proposition is equivalent to the statement that two cohomologous forms on $V$ which are nondegenerate on $\Gamma \cup F$ are isotopic $(\operatorname{rel} \Gamma \cup F)$.

Proof. We claim that there is a family of symplectically embedded curves $\Gamma_{t}$ which joins $\Gamma_{0}$ to $\Gamma_{1}$. Using this, one easily finds a suitable family $g_{t}$ of embeddings $\left(\Gamma_{0}, \Gamma_{0} \cap F\right) \rightarrow(V, F)$ such that $g_{1}\left(\Gamma_{0}\right)=\Gamma_{1}$, which may be extended to $V$ by the symplectic isotopy extension theorem. Clearly, it suffices to consider the case when one of the sections, say $\Gamma_{0}$, is standard. As in Lemma 4.16 , this means that it is $J_{k}$-holomorphic for some complex structure $J_{k}$ on $V \equiv P\left(L_{k} \oplus \mathbb{C}\right)$ where $k=D \cdot D$ and $D=\left[\Gamma_{0}\right]$. One can construct the family $\Gamma_{t}$ as the set of $J_{t}$-holomorphic $D$-curves through a generic path $\beta(t)$ in the $m$-fold product $V \times \cdots \times V$, where $m$ is chosen so that there is exactly one $J_{t}$-holomorphic $D$-curve through each generic $m$-tuple of distinct points. The details of the argument are very similar to those of Lemma 4.16, and will be left to the reader.

\section{THE ClASSIFICATION THEOREMS}

Our first aim is to classify minimal rational pairs $(V, C, \omega)$. It will be convenient to amplify the definition of "simple" given in (2.2) to take into account that in dimension 4 the connectedness of a cusp-curve can be described homologically. Thus we will say that the class $A$ is $J$-simple if it cannot be written as a sum $A_{1}+\cdots+A_{m}$ for some $m \geq 2$ where

(i) each $A_{i}$ has a $J$-holomorphic representative $S_{i}$; and

(ii) if $A_{i} \neq A_{j}$ there is a sequence $A_{i}=A_{i_{1}}, A_{i_{2}}, \ldots, A_{\text {in }}=A_{j}$ such that $A_{i p} \cdot A_{i p+1} \geq 1$ for $p=1, \ldots, n-1$.

It is easy to see that these conditions are satisfied if the $A_{i}$ are the classes of the components of any $A$-cusp-curve. Further, condition (ii) is phrased in such a way that the class $m B, m \geq 2$, is not $J$-simple if $B$ has a $J$-holomorphic representative, since it has the allowable decomposition $B+\cdots+B$.

Let $C$ be a symplectically embedded 2 -sphere with $C \cdot C \geq 0$.

Lemma 5.1. There is an element $J$ of $\mathscr{U}_{\infty}$ such that the class [C] may be represented by a $J$-holomorphic cusp-curve $S=S_{1} \cup \cdots \cup S_{m}$, where, for each $i$, the class $A_{i}=\left[S_{i}\right]$ is $J$-simple and $J$ is regular for $A_{i}$-curves. 
Proof. Let $J_{0}$ be an $\omega$-tame almost complex structure for which $C$ has a $J_{0}$-holomorphic parametrization. As in the proof of Proposition 4.1, we may assume that $J_{0}$ is a regular value for the projection operator $P_{C}$ and that it belongs to $\mathscr{U}_{\infty}$. It follows easily from the compactness theorem that there is a neighbourhood $N\left(J_{0}\right)$ of $J_{0}$ in $\mathscr{J}(\omega)$ such that only finitely many classes $A$ in $H_{2}(V ; \mathbb{Z})$ with $\omega(A) \leq \omega(C)$ have $J$-holomorphic representatives for some $J$ in $N\left(J_{0}\right)$. Hence we may further assume that $J_{0}$ is a regular value for all such $P_{A}$.

Thus there are only finitely many decompositions of the homology class $[C]$ as $A_{1}+\cdots+A_{m}$ which satisfy the conditions (i) and (ii) above with $J=J_{0}$. Clearly, each such decomposition gives rise either to a cusp-curve $S=S_{1} \cup$ $\cdots \cup S_{m}$ or to a representation of $[C]$ as a multiply-covered curve. Corollary 2.10 implies that in the latter case $[C]$ has a decomposition with $m=2$, $A_{1}=A_{2}$, and $A_{1} \cdot A_{2}=1$, so that it also has a representation by a cusp-curve. Thus, if $[C]$ is not $J_{0}$-simple, it may always be represented by a cusp-curve. If the constituent components of this cusp-curve are not simple, they may be decomposed further. And so, among the finite set of decompositions of $[C]$, there clearly is at least one such that each $A_{i}$ is $J_{0}$-simple.

Lemma 5.2. Let $J$ be a regular value for $P_{A}$ and suppose that $A$ is a $J$-simple class which may be represented by an embedded J-holomorphic 2-sphere. Then $p=A \cdot A \leq 1$.

Proof. Since $A$ can be represented by an embedded curve, $c(A)=2+A \cdot A$ and the dimension of the compact manifold $M(J, A)=M_{p}(J, A) / G$ is $2 c(A)-2=$ $2 p+2$.

Suppose that $p>1$ and let $\mathscr{M}_{A}$ be the set of pairs $(f, J)$ where $J \in \mathscr{J}(\omega)$ and $f$ is $J$-holomorphic. Then consider the evaluation map

$$
e_{A, p}: \mathscr{M}_{A} \times_{G}\left(S^{2} \times \cdots \times S^{2}\right) \rightarrow V \times \cdots \times V
$$

given by $\left(f, J, z_{0}, \ldots, z_{p}\right) \mapsto\left(f\left(z_{0}\right), \ldots, f\left(z_{p}\right)\right)$, where there are $p+1$ factors in each product and $G$ acts diagonally. We first claim that $e_{A, p}$ is transverse to the inclusion $j: V \rightarrow V \times \cdots \times V$ given by $z \mapsto\left(z, x_{1}, \ldots, x_{p}\right)$, where $x_{1}, \ldots, x_{p}$ are distinct points. To prove this, it suffices to show that given tangent vectors $v_{i}$ to $V$ at $x_{i}$ for $1 \leq i \leq p$, there are a family of diffeomorphisms $g_{t}$, almost complex structures $J_{t}$, and a tangent vector $w_{0}$ at $x_{0}$ so that the path $e_{A, p}\left(g_{t} \circ f, J_{t}, z_{0}, z_{1}, \ldots, z_{p}\right)$ is tangent to $\left(w_{0}, v_{1}, \ldots, v_{p}\right)$ at $t=0$. But this is obvious if $x_{0} \neq$ any $x_{i}$, and it holds if $x_{0}=x_{i}$ for some $i$ since we may take $w_{0}=v_{i}$. Therefore, because $P_{A}$ is a Fredholm map from the manifold $e_{A, p}^{-1}(\operatorname{Im} j)$ to $\mathscr{J}(\omega)$,

$$
R=P_{A}^{-1}(J) \cap e_{A, p}^{-1}(\operatorname{Im} j)
$$

is a manifold for generic $J$. It is easy to check that $R$ is a compact 4-manifold and that the composite $e=\operatorname{pr} \circ e_{A, k}$ is a degree 1 map from $R$ onto $V$, where $\mathrm{pr}$ is the projection onto the first factor. 
Let $\pi: M_{p}(J, A) \times{ }_{G}\left(S^{2} \times \cdots \times S^{2}\right) \rightarrow M_{p}(J, A) / G$ be the obvious projection. We claim that $\pi$ induces a submersion of $R$ onto a submanifold $\pi(R)$ of $M_{p}(J, A) / G$. In fact, if $p \geq 3$, then, for each $C \in \pi(R)$, there is a unique parametrization $f_{C}$ of $C$ which takes three fixed points $w_{1}, w_{2}, w_{3}$ to the three points $x_{1}, x_{2}, x_{3}$. Hence, $R \cap \pi^{-1}(C)=\left\{\left(f_{C}, z, w_{1}, \ldots, w_{p}\right): z \in\right.$ $\left.S^{2}\right\}$, where $f_{C}^{-1}\left(x_{i}\right)=w_{i}$ for $3<i \leq p$. Thus, in this case, $R$ is diffeomorphic to the product of $S^{2}$ with the manifold $\pi(R)$. When $p=2$, one can find a local product structure near a curve $C_{0} \in \pi(R)$ as follows. Choose a third point $x_{3}$ on $C_{0}$ and let $T \subset V$ be a little 2-disc which is transverse to $C_{0}$ at $x_{3}$. Then choose the parametrization $f_{C}$ of the curves $C \in \pi(R)$ near $C_{0}$ by requiring that $f\left(w_{i}\right)=x_{i}$ for $i=1,2$ and $f\left(w_{3}\right) \in T$. Since $f_{C}$ is unique, the previous argument shows that $\pi: R \rightarrow \pi(R)$ is a locally trivial fibration.

We now claim that $\pi(R) \cong S^{2}$. To see this, identify the tangent space to $V$ at $x_{1}$ with $\mathbb{C}^{2}$ and consider the map $R \rightarrow \mathbb{C} P^{1}=S^{2}$ given by

$$
\left(f, z_{0}, \ldots, z_{p}\right) \rightarrow \text { tangent space to } \operatorname{Im} f \text { at } f\left(z_{1}\right)=x_{1} \text {. }
$$

This map is well defined since all the elements of $R$ are embeddings by (2.6). Further, it clearly factors through $\pi$, so that we get a map $\theta: \pi(R) \rightarrow S^{2}$. It follows easily from (2.5) that this map is injective. Hence it must be a homeomorphism.

Thus $R$ is an $S^{2}$-bundle over $S^{2}$, and $e: R \rightarrow V$ is a degree 1 map which takes the fiber $F$ of $R$ to a curve in class $A$. Since $F \cdot F=0$ and $A \cdot A=p>1, e$ cannot induce an isomorphism on homology and cohomology. However, it does induce an injection on cohomology since dege $=1$. It follows that the cohomology ring of $V$ is isomorphic to that of $\mathbb{C} P^{2}$. Since there is no injection of $H^{*}\left(\mathbb{C} P^{2} ; \mathbb{Z}\right)$ into $H^{*}\left(S^{2} \times S^{2} ; \mathbb{Z}\right)$ which is an isomorphism on $H^{4}$, the bundle $R$ must be nontrivial. An easy calculation now shows that, if $p>1$, there is no suitable injection in this case either.

Lemma 5.3. Let $(V, C, \omega)$ be minimal, and suppose that $C$ is a rational curve with $C \cdot C \geq 0$.

(i) We may assume in Lemma 5.1 that the $S_{i}$ are distinct embedded curves with self-intersection numbers equal to $-1,0$, or 1 .

(ii) If $S_{i} \cdot S_{i}=1$ for some $i$, then $V=\mathbb{C} P^{2}$ and $m=1$ or 2 .

(iii) If $S_{i} \cdot S_{i}=-1$ for some $i$, then there is only one such $i$ and all the other $S_{j}$ are homologous and have $S_{j} \cdot S_{j}=0$. Further $V=\mathbb{C} P^{2} \# \overline{\mathbb{C P}}^{2}$.

(iv) If $S_{i} \cdot S_{i}=0$ for all $i$, then all the $S_{i}$, except perhaps for one, are homologous, and $V$ is an $S^{2}$-bundle.

Proof. (i) Let $J$ be as in Lemma 5.1. Because $J \in \mathscr{U}_{\infty}$, each $c\left(A_{i}\right) \geq 1$, and so the cusp-curve $S$ satisfies the conditions of Proposition 2.11. Using the minimality of $(V, C, \omega)$ and Proposition 2.11(iii), one sees that the only components $S_{i}$ with negative self-intersection are disjoint exceptional curves. 
Therefore, all the components of $S$ are embedded and disjoint except possibly for those which are $k$-fold covers of embedded curves $T$ with $T \cdot T=0$. However, as explained in the proof of Proposition 2.11, we may replace such components by $k$ disjoint copies of $T$. The result now follows from Lemma 5.2 .

(ii) We showed in Lemma 3.2(a) of [McD2] that if $V$ contains a $J$-simple class which has an embedded representative of self-intersection 1 , then $V=$ $\mathbb{C} P^{2}$. The fact that $m \leq 2$ follows by Proposition 2.11(i).

(iii) Now suppose that for some $r \geq 1$ the curves $S_{1}, \ldots, S_{r}$ have selfintersection -1 . By (i) and (ii) the other $S_{i}$ have self-intersection 0 . If the $S_{i}$ for $i>r$ represent at least two homology classes, say $B_{1}$ and $B_{2}$, then, by the argument of Proposition 4.1, each point of $V$ lies on a unique ( $J$-holomorphic) embedded $B_{1}$-curve and a unique ( $J$-holomorphic) embedded $B_{2}$-curve. Since each $B_{1}$-curve intersects each $B_{2}$-curve exactly once, $V$ must be diffeomorphic to $S^{2} \times S^{2}$. But this is impossible since $r>0$.

Thus $[C]=A_{1}+\cdots+A_{r}+k B$ where the $A_{i}$ are distinct and $k=m-r \geq 0$. We aim to show that $r=1$ and $k \geq 1$. Suppose first that $k=0$. Then, by minimality, $A_{i} \cdot[C] \geq 1$ for all $i$. Hence $C \cdot C \geq r=c(C)$, which contradicts the fact that $C \cdot C=c(C)-2$. On the other hand, if $k \geq 1$, then $V$ is an $S^{2}$-bundle over a Riemann surface $M$. The only such bundle which contains a 2-sphere with self-intersection -1 is the nontrivial bundle over $S^{2}$, and this contains just one homology class of such spheres. Therefore $r=1$ as claimed.

(iv) We saw in (iii) above that if the $S_{i}$ represent more than one distinct class with $A_{i} \cdot A_{i}=0$, then $V$ must be $S^{2} \times S^{2}$. Hence there can be at most two distinct classes. Further, if $C=m_{1} A_{1}+m_{2} A_{2}$ satisfies $C \cdot C=c(C)-2$ then at least one of $m_{1}$ and $m_{2}$ must be $\leq 1$.

(5.4) Proof of Theorem 1.4 and Corollary 1.5. The statements about the diffeomorphism type of $(V, C)$ follow immediately from Lemma 5.3. When $V=\mathbb{C} P^{2}, V$ contains an embedded curve of self-intersection 1 by Lemma 5.3(i) and so has the standard Kähler structure by [G, 2.4. $\left.\mathrm{B}_{3}^{\prime}\right]$; see also [McD2, Note 4.2]. The claims made about symplectic structures on $S^{2}$-bundles are proved in $\S 4$. Further, if $C \cdot C=0$, the symplectomorphism from $V$ to the bundle may be assumed to take $C$ to a fiber by Proposition 4.1.

If $C \cdot C \geq 2$ then we are in case (iii) or (iv) of Lemma 5.3. In case (iii), $[C]=E+k A$, where $E \cdot E=-1$ and $A \cdot A=0$. Since $C$ is connected $E \cdot A>0$. In fact, $E \cdot A$ must be 1 , because $C \cdot C=c(C)-2$. Hence $[C] \cdot A=1$ and $C$ meets each fiber exactly once. Thus $C$ is a section of the nontrivial bundle $\pi: V \rightarrow S^{2}$, and the desired uniqueness statement is proved in Proposition 4.18. Similar remarks apply in case (iv). 
(5.5) Proof of Theorem 1.2. We must show that the category of symplectic manifolds $(V, \omega)$, which contain a symplectically embedded 2 -sphere $C$ of nonnegative self-intersection, is closed under blowing up and down, and under perturbations of $\omega$ through noncohomologous forms. Let us first consider what happens when we blow down the curve $\Sigma$. We first claim that by isotoping $C$ (or equivalently $\Sigma$ ) we may assume that both $C$ and $\Sigma$ are $J$-holomorphic for some $J$ which is integrable near $\Sigma$. This follows by choosing $J \in \mathscr{U}_{\infty}$ as in Proposition 4.1 so that it is integrable near $C$ and $C$ is $J$-holomorphic; isotoping $\Sigma$ to the $J$-holomorphic representative of $[\Sigma]$ as in $(3 \cdot 3)$; and then making $J$ integrable near $\Sigma$. By Lemma 3.2 we may suppose that the blowingdown map $V \rightarrow \bar{V}$ is $J-\bar{J}$-holomorphic for a suitable almost complex structure $\bar{J}$ on $\bar{V}$. Hence $C$ pushes forward to an embedded $\bar{J}$-holomorphic (and hence symplectic) curve in $(\bar{V}, \bar{\omega})$, the self-intersection number of which is equal to $C \cdot C+C \cdot \Sigma \geq C \cdot C$.

Next, consider blowing up. By Theorem 1.1 it suffices to consider the case when $(V, C, \omega)$ is a minimal pair which is being blown up at $k$ points. Thus we are cutting out the images $g\left(\coprod_{i} B\left(\lambda_{i}\right)\right)$ of $k$ balls in $V$ and collapsing their boundaries to exceptional curves. Clearly, a symplectically embedded copy of $S^{2}$ in $V$ which is disjoint from $g\left(\coprod_{i} B\left(\lambda_{i}\right)\right)$ will lift to the blow up $\left(V^{\prime}, \omega^{\prime}\right)$. If the $\lambda_{i}$ are large, it is not obvious that this will exist. However, by reducing the $\lambda_{i}$ one can clearly find a family of symplectic forms $\omega_{t}^{\prime}$ starting at $\omega^{\prime}$ and such that $\left(V^{\prime}, \omega_{1}^{\prime}\right)$ does contain a symplectically embedded copy $C^{\prime}$ of $S^{2}$. Moreover, by Lemmas 5.1 and 5.2 , we may suppose that $C^{\prime} \cdot C^{\prime}=0$ or 1 . When $C^{\prime} \cdot C^{\prime}=1$, one can now complete the argument as in Lemma 4.10 , the only difference in the present situation being that there are several classes $E_{i}$ which may be represented by exceptional curves instead of just one. When $C^{\prime} \cdot C^{\prime}=0$ one argues similarly, noting that, by Proposition 2.11 , if $J \in \mathscr{U}$ the only $J$-holomorphic $\left[C^{\prime}\right]$-cusp-curves consist of a pair of intersecting exceptional curves.

The proof of the deformation result is similar and will be left to the reader.

We now turn to Theorem 1.7, which concerns the filling of lens spaces. In view of the above results, it clearly suffices to prove the following lemma.

Lemma 5.6. (i) Equivalence classes of symplectic fillings of $\left(L_{p}, \beta\right)$ correspond to equivalence classes of triples $\left(V, C_{p}, \omega\right)$ where $C_{p}$ is a symplectically embedded 2-sphere with $C_{p} \cdot C_{p}=p$ in the closed symplectic manifold $(V, \omega)$.

(ii) Minimal fillings correspond to $\Lambda$-minimal triples $\left(V, C_{p}, \omega\right)$, where $\Lambda=$ $\left\{A: A \cdot\left[C_{p}\right]=0\right\}$.

Note. The equivalence relation in (i) can either be that of diffeomorphism or that of symplectomorphism.

Proof. Let $\left(N_{p}, \sigma\right)$ be the quotient by $\Gamma_{p}$ of a small neighbourhood of $\partial B^{4}(1)$ in $B^{4}(1)$. Further, let $U_{p}$ be a small neighbourhood of the zero section $S_{p}$ in a 
complex line bundle over $\mathbb{C} P^{1}$ with $c_{1}=p$. By the symplectic neighbourhood theorem, $U_{p}$ has a symplectic form $\tau$ which is unique up to isotopy $\left(\operatorname{rel} S_{p}\right)$ and which restricts on $S_{p}$ to the standard form with integral $\pi$. It is not hard to check that there is a continuous map

$$
\Psi:\left(N_{p}, L_{p}, \sigma\right) \rightarrow\left(U_{p}, S_{p}, \tau\right)
$$

such that $\Psi^{*}(\tau)$ is well defined and equal to $\sigma$. In fact, using the obvious coordinates, we may take

$$
\begin{aligned}
\Psi\left(z_{1}, z_{2}\right) & =-\left(1 / r^{2}-1\right)^{1 / 2} \cdot\left(z_{1}, z_{2}\right) \quad \text { if } r^{2}=\left|z_{1}\right|^{2}+\left|z_{2}\right|^{2}<1, \\
& =\left[z_{1}: z_{2}\right] \in \mathbb{C} P^{1} \quad \text { if } r^{2}=1 .
\end{aligned}
$$

Thus $\Psi$ is a diffeomorphism except on $L_{p}$, and restricts on $L_{p}$ to the standard circle fibration $L_{p} \rightarrow S_{p}$. Hence, if $(Z, \omega)$ is a filling of $\left(L_{p}, \sigma\right)$, we can replace a neighbourhood of $L_{p}$ by a neighbourhood of $S_{p}$ thus getting a triple $\left(V, C_{p}, \omega\right)$, and conversely. The rest of the lemma is obvious.

\section{ACKNOWLEDGMENTS}

I wish to thank Ya. Eliashberg for many stimulating discussions about the questions studied here, and for encouraging me to write this paper. I am also grateful to M. Audin for pointing out some inaccuracies in $\S 5$, and to MRSI for its hospitality and support. A summary of the results presented here has appeared in [McD6]. Some of the material in this paper originally appeared in the preprint Asymptotically flat symplectic manifolds.

\section{REFERENCES}

[Au] M. Audin, Hamiltoniens périodiques sur les variétés symplectiques compactes de dimension 4, preprint, IRMA, Strasbourg, 1988.

[E1] Ya. Eliashberg, On symplectic manifolds which are bounded by standard contact spheres, and exotic contact structures of dimension > 3, J. Differential Geom. (to appear).

[E2] - private communication.

[GH] Ph. Griffiths and J. Harris, Principles of algebraic geometry, Wiley, New York, 1978.

[G] M. Gromov, Pseudo-holomorphic curves in symplectic manifolds, Invent. Math. 82 (1985), 307-347.

[GL] V. Guillemin and E. Lerman, Fiber bundles with symplectic fibers, preprint, MIT, Cambridge, MA, 1989.

[GS] V. Guillemin and S. Sternberg, Birational equivalence in the symplectic category, Invent. Math. 97 (1989), 485-522.

[McD1] D. McDuff, Examples of symplectic structures, Invent. Math. 89 (1987), 13-36.

[McD2] __ Blowing up and symplectic embeddings in dimension 4, Topology (to appear).

[McD3] _ Elliptic methods in symplectic geometry, Bull. Amer. Math. Soc. (to appear).

[McD4] _ The local behaviour of holomorphic curves in almost complex 4-manifolds, preprint, SUNY, Stony Brook, NY, 1989.

[McD5] _ Symplectic manifolds with contact-type boundaries, preprint, 1990.

[McD6] __ Rational and ruled symplectic 4-manifolds, Proc. Conf. at Durham, Summer 1989 (S. Donaldson and C. Thomas, eds.), Pitman, Oxford (to appear). 
[W] J. Wolfson, Gromov's compactness of pseudo-holomorphic curves and symplectic geometry, J. Differential Geom. 28 (1988), 383-405.

ABSTRACT. This paper investigates the structure of compact symplectic 4-manifolds $(V, \omega)$ which contain a symplectically embedded copy $C$ of $S^{2}$ with nonnegative self-intersection number. Such a pair $(V, C, \omega)$ is called minimal if, in addition, the open manifold $V-C$ contains no exceptional curves (i.e., symplectically embedded 2 -spheres with self-intersection -1$)$. We show that every such pair $(V, C, \omega)$ covers a minimal pair $(\bar{V}, C, \bar{\omega})$ which may be obtained from $V$ by blowing down a finite number of disjoint exceptional curves in $V-C$. Further, the family of manifold pairs $(V, C, \omega)$ under consideration is closed under blowing up and down. We next give a complete list of the possible minimal pairs. We show that $\bar{V}$ is symplectomorphic either to $\mathbb{C} P^{2}$ with its standard form, or to an $S^{2}$-bundle over a compact surface with a symplectic structure which is uniquely determined by its cohomology class. Moreover, this symplectomorphism may be chosen so that it takes $C$ either to a complex line or quadric in $\mathbb{C} P^{2}$, or, in the case when $\bar{V}$ is a bundle, to a fiber or section of the bundle.

Department of Mathematics, State University of New York at Stony Brook, Stony BROOK, NEW YORK 11794-3651

E-mail address: dusa@math.sunysb.edu 